\title{
The Amyloid Precursor Protein regulates human cortical neurogenesis
}

Khadijeh Shabani ${ }^{1}$, Julien Pigeon ${ }^{1}$, Marwan Benaissa Touil Zariouh ${ }^{1}$, Tengyuan Liu ${ }^{1,2}$, Azadeh Saffarian $^{3}$, Jun Komatsu ${ }^{3}$, Elise Liu ${ }^{1}$, Natalia Danda ${ }^{1}$, Ridha Limame ${ }^{1}$, Delphine Bohl ${ }^{1}$, Carlos Parras $^{1}$, Bassem A. Hassan ${ }^{1 *}$.

${ }^{1}$ Institut du Cerveau - Paris Brain Institute - ICM, Sorbonne Université, Inserm, CNRS, Hôpital Pitié-Salpêtrière, Paris, France.

${ }^{2}$ Doctoral School of Biomedical Sciences, KU Leuven, Leuven, Belgium

${ }^{3}$ Scipio bioscience, iPEPS-ICM, Hôpital Pitié-Salpêtrière, Paris, France

*Correspondence to: bassem.hassan@icm-institute.org.

\section{Abstract}

The human neocortex has undergone significant expansion during evolution partially underlying increased human cognitive capacities. The 16 billion neurons of the human neocortex are derived from a limited number of cortical neural progenitor cells (NPCs). Human cortical NPCs initially generate neurons at a slow rate while preserving their progenitor state for a prolonged period, partly contributing to increased human cortical size. How the balance between the progenitor state and neurogenic state is regulated, and whether it contributes to species-specific brain patterning, is poorly understood. We find that the human Amyloid Precursor Protein (APP), whose mutations cause Alzheimer's disease, specifically regulates this fine balance. Mechanistically, APP regulates these two aspects via two pathways: the AP1 transcription factor and the canonical Wnt pathway. We propose that APP is a homeostatic regulator of the neurogenic potential of cortical NPCs thus potentially contributing to human-specific patterns of neurogenesis.

\section{Introduction}

Human cortical neurogenesis is partly distinguished by human NPCs retaining a self-renewing progenitor state for a particularly long time presumably through species-specific mechanisms that remain poorly understood. The highly conserved class I transmembrane Amyloid precursor protein (APP) is broadly expressed throughout nervous system development and has been extensively studied because its proteolytic processing is linked to Alzheimer's disease $\mathrm{AD}^{1,2,3}$, yet its physiological function, especially in humans, is unclear. APP and its homologues in various species are involved in many biological processes such as axonal outgrowth after injury ${ }^{4}$, endolysosomal pathway, ${ }^{5,6,7}$, stress response after hypoxia/ischemia ${ }^{8,9}$, cell signaling processes and brain development and plasticity ${ }^{10,11}$. In the worm, C. elegans loss of the APP homologue Apl is lethal due to molting deficits, while causing mild and low penetrance defects in various aspects of neuronal differentiation, function, and survival in mouse and Drosophila ${ }^{12}$. In humans, point 
mutations of even a single copy of $A P P$ cause familial $\mathrm{AD}^{13}$. Furthermore, genetic loss of function data obtained from the human population by the GENOMAD project show that APP has a loss of function observed/expected (LOEUF) score of 0.42, meaning that complete loss of APP causes $>50 \%$ developmental mortality, a rate surprisingly higher than that of key neurodevelopmental genes such as Neurog2 $(\mathrm{LOEUF}=0.54)$ and Ascl1 $(\mathrm{LOEUF}=0.59)^{14,15}$. Furthermore, a clinical case of a homozygous nonsense truncating mutation in APP leading to microcephaly has been reported $^{16,17}$, indicating that human development may be particularly sensitive to the loss of $A P P$. APP is highly expressed in human telencephalic neurospheres, and during the differentiation and migration of cortical neurons ${ }^{18,19,20}$ suggesting that it may be involved in NPC proliferation, differentiation and/or maturation ${ }^{21,22}$. In the adult rodent brain, APP is also abundantly expressed in NPCs found in the VZ-SVZ ${ }^{23,24}$, but it's loss does not cause obvious deficits in neurogenesis. We generated $A P P$-knockout iPSCs in two different genetic backgrounds and queried the potential effect on human cortical neurogenesis.

\section{Results}

\section{Loss of APP accelerates neurogenesis of cortical NPCs}

We generated $A P P-K O$ iPSC by CRISPR/Cas9 ( ig. S1, A to K) and chose three $A P P-K O$ clones and one $A P P$ wild type (control) clone, which received Cas9 but was not mutated, for further analysis. Following induction of cortical differentiation, the morphology and molecular identity of NPCs was normal in all clones (fig. S2). Seven days after initiation of neuronal differentiation, we observed many more cells with neuronal-like morphology in APP-KO NPC than in control (fig. S3, A and B). We stained the cells for SOX2 (NPCs), Doublecortin (DCX; immature neurons), $\beta$ TubulinIII (TUJ1; neurons) to determine cell type. All three $A P P-K O$ clones showed increased TUJ1 and DCX expression 7 days post differentiation (Fig. 1, A to H and fig. S3, C to J). To determine the neurogenic state of control and $A P P-K O$ NPCs, we sparsely labelled them (70 control NPCs and an average of 63.6 NPCs in the 3 KO lines, Supplementary Table 1) with a GFPexpressing lentiviral vector ${ }^{25,26}$ (Fig. 1I) and quantified the number of GFP+ NPCs (SOX2) and neurons (TUJ1) at days 0,7 and 30 after differentiation (Fig. 1, J to S and fig. S3, I-R). We observed significant decrease in NPCs (Fig. 1T) accompanied by significantly more production of neurons over time (Fig. 1U) in all three $A P P-K O$ compared to control after 7 days. The neuron-toprogenitor ratio was strikingly higher in all 3 APP-KO lines compared to controls at day 7, but 
was not significantly different by day 30 . Focusing on this initial burst of neurogenesis in $A P P$ $K O$ NPCs, we found that 1 control NPC produced on average 8 NPCs and 3.6 neurons after 7 days of differentiation. In contrast, within the same time frame each APP-KO NPC produced on average 1.25 NPCs and 22.9 neurons (Supplementary Table 1), indicating a major switch of cortical NPCs from a self-amplifying state to neurogenic state.

APP is broadly expressed in the developing nervous system. In order to examine whether this effect is general or brain-region specific, we generated motor neurons ${ }^{27}$ from control and $A P P$ KO2 iPSCs. We confirmed the expression of APP protein in motor neuron precursor cells (fig. S4A) and examined them at 1 and 4 days post-differentiation. No significant differences were detected in phase contrast morphology (fig. S4, B to E) or the number of ISLET1+ cells (MN marker) in $A P P-K O 2$ and control (fig. S4, F-R) at either time point. These data suggest that loss of APP preferentially affects cortical progenitors and causes either an increased neurogenic state of cortical NPCs or accelerated differentiation of their daughters.

\section{Loss of APP does not alter the order or timing of cortical neuron differentiation}

One of the key features of cortical development is the sequential generation of different neuronal subtypes that will occupy different layers of the neocortex. This feature is preserved in NPC cultures, whereby deep layer (early born) neuronal fate markers appear before upper layer (late born) cell fate markers ${ }^{28,29}$. To assess whether loss of APP affects this order, differentiation was launched and cells were tracked for 7, 15, 30, 60- and 120-days post-differentiation. We first observed clear expression of early born neuronal marker CTIP2 only at day 15 in both genotypes, despite the fact that $A P P-K O$ NPCs had generated $\sim 3$-fold more neurons than control NPCs (Fig. 2, A1-F4). Within 30 days, the number of CTIP2+ and STAB2+ cells increased in both control and $A P P-K O 2$ (Fig. 2, G1-H4). The same results were observed for $A P P-K O 1$ and $A P P-K O 3$ at 7 , 15- and 30-days post-differentiation (data not shown). We next focused on markers of neuronal maturation namely the emergence of synapse markers 60 and 120 days post-differentiation. MUNC13 (presynaptic) was first detectable 60 days post-differentiation in both control and APP$K O$ while no PSD95 (postsynaptic) was detected in both (fig. S5, A to L). MUNC13, PSD95 and HOMER1 (glutamatergic synapses) were clearly detectable 120 days post-differentiation in control and $A P P-K O$ s (fig. S6, A to $\mathrm{H}$ ). These data suggest that loss of APP does not significantly accelerate maturation of new born neurons, and hint that the deficit is in the NPCs themselves. 


\section{APP knockout NPCs are in an elevated neurogenic state}

Temporal analysis of APP expression (http://www.humous.org) in scRNAseq data from the developing human fetal cortex ${ }^{30}$ shows higher expression levels in early NPCs, when selfamplifying divisions dominate, and a decrease over time as NPCs switch to neurogenic divisions. To unravel the earliest detectable defect in the absence of APP, NPCs we were maintained under progenitor conditions without induction of differentiation. We confirmed APP expression in control NPCs by western blot (fig. S7, A), and stained for SOX2/ TUJ1 and SOX2/ DCX (Fig. 3, A to L and fig. S7, B to M). We observed a significant decrease in the ratio of SOX2+ cells (Fig. $3 \mathrm{M}$ and fig. S7N) and significant increase in the ratio of DCX+ cells (Fig. 3N and fig. S7O) in $A P P-K O$ s relative to control, even in the absence of induction of differentiation. This was accompanied by a significant reduction in proliferative NPCs in APP-KOs compared to controls ( $20 \%$ fewer Ki67+ cells; Fig. 3, O to Q and fig. S7, P to R). These data suggest that APP is required to maintain human cortical NPCs in a self-renewing progenitor state by inhibiting premature neurogenesis. To rule out genetic background effects, we generated an $A P P-K O$ clone from a different independent iPSC line (fig. S1, L to N) and observed the same results in those NPCs (fig. S7, S to Z). We performed a rescue experiment by transfecting APP-KO2 NPCs with PiggyBac vectors containing either GFP alone ( $p P B-C A G-I R E S-E G F P)$ or GFP and APP ( $p P B-$ CAG-hAPP-IRES-EGFP) and staining for SOX2 and DCX. Quantification of GFP+/SOX2+ and $\mathrm{GFP}+/ \mathrm{DCX}+$ cells showed that $A P P-K O \mathrm{NPCs}$ were restored to a progenitor state and the number of their neuronal progeny was reduced back to control levels upon rescue with APP (Fig. 3, R1 to V), but not with GFP alone. We noted that restoring APP only rescued NPCs expressing it, but not neighboring cells (arrows in Fig. 3T2), suggesting a cell-autonomous requirement. Next, we produced cortical organoids from control and $A P P-K O$ clones from the two independent iPSC lines and stained them for SOX2 and DCX at day 15, when the majority of cells are in the NPC state. We observed larger DCX+ clusters with higher levels of DCX expression in APP-KO organoids of both iPSC lines (fig. S7, Z'1 to Z'8 iPSC line1 and Z'1 to Z"8 iPSC line 2). Finally, to test whether this effect on cortical neurogenesis was specific to human NPCs, we tested for signs of premature neuronal differentiation in the $A P P-K O$ mouse cortex at a stage when most cells should still be progenitors. Previous work on cultured mouse NPCs reported contradictory observations with respect to the effect of loss of APP on neuronal differentiation ${ }^{31,32}$ but the most comprehensive study reported no evidence for a role of APP or its paralogues in mouse neurogenesis in vitro or 
in vivo ${ }^{33}$. We examined the developing mouse cortex at E10.5 and observed no Tuj $1+$ cells in the progenitor layer and no difference in Pax6 (NPC) and Tuj1 expression between App $+/+$ and App/- brains (fig. S8, A-J). Together, these data show that APP is cell autonomously required to repress neurogenic divisions of human cortical NPCs.

\section{APP knockout NPCs are on an accelerated trajectory of differentiation}

To gain insight into how APP regulates the fate of human cortical NPCs, we performed single cell RNA sequencing using a novel, non-microfluidics-based method, on control and $A P P-K O$ NPCs (Supplementary Table 2, BioProject accession number PRJNA678443). Cluster analysis identified five clusters in control NPCs and seven clusters in APP-KO NPC ( $A P P-K O 2)$ that presented very close relationship between genotypes and lineage progression stages as shown by uniform manifold approximation and projection (UMAP) 2D-representation (Fig. 4A and fig. S9, A to C). We used the main marker genes of each cluster to generate heat maps, and based on these markers, the five clusters of control NPCs were annotated as radial glial cells (RGC), cycling progenitors 1 and 2 (CP1 and CP2), intermediate progenitors (IP) and neurons (N) (fig. S9, D to F). These same five categories were also found in $A P P-K O$ together with two additional clusters. One cluster, which we refer to as "neurogenic progenitors" (NPs), is composed of cells expressing both radial glial markers (e.g. FABP7 and VIM) as well as neuronal differentiation markers (e.g. PNT, SPARC, and TUBB2A; Supplementary Table 2). In addition, we found a very small cluster of cells ( $3 \%$; "other") enriched in pericyte markers, while the neuronal cluster represented twice as many cells (5\% vs $2 \%$ ) in the $A P P-K O$ sample. Interestingly, NPs represent $13 \%$ of $A P P-K O$ cells and together with the neurons appear at the expense of cycling progenitors, which show a reduction of $20 \%$ in APP-KO cells compared to control (Fig. 4B), matching the reduction we observed with Ki67 immunofluorescence (Fig. 3Q). Furthermore, pseudotime analysis shows a clear temporal shift towards a differentiated state in all $A P P-K O$ clusters compared to similar control clusters (Fig. 4, C and D). To further test these observations, we performed SOX2/NEUROG2/DCX triple staining (Fig. 4, E to L) on control and $A P P-K O$ NPCs. NEUROG2 expression marks commitment to differentiation of mammalian NPCs ${ }^{34,35}$. We observed a $\sim 15$-fold increase in the number of NEUROG2+ NPCs (SOX2+/NEUROG2+; Fig. 4, I and K) in APP-KO compared to control (Fig. $4 \mathrm{M})$. This closely mirrors the ratio of neurons-to-progenitors ( $\sim 18$-fold) we found to be produced by $A P P-K O$ NPCs. 


\section{Loss of APP triggers the immediate early gene response to drive cortical neurogenesis}

To gain insight into the mechanisms of the gain of neurogenic state in the absence of APP, we performed bulk RNA sequencing in $A P P-K O$ and control NPCs. We observed only 763 differentially expressed genes (558 up; 205 down including $A P P$ as expected) between control and $A P P-K O 2$ NPC (Supplementary Table 3 and fig. S10, A and B) indicating relative specificty. Consistent with the $A P P-K O$ phenotype, bulk RNAseq showed downregulation of primary progenitor's markers $P A X 6$ and $C D O N$, and upregulation of neurogenic genes NEUROG2, BCL6, $A S C L 1, N H L H 1$, and $D C X$. Moreover, $C D K N 2 B$ a well-known cell cycle inhibitor and NPC differentiation promotors $D L L 3, J A G 2$, and DNER were also upregulated (Fig. 4N). One pathway whose activity is intimately linked to APP function in a variety of organisms and neuronal contexts is the JUN N-terminal Kinase (JNK) pathway ${ }^{36}$ whose effector proteins, JUN and FOS, combine to form the AP1 transcription factor. Evidence supports APP-dependent transcriptional repression of c-Jun and reduced basal c-Jun N-terminal kinase (JNK) activity in PC12 cells ${ }^{37}$ and activation of the JNK signaling pathway were reduced either by over expression of APP or treatment with sAPP $\alpha^{37-39}$. Moreover, $c-F o s$ expression is upregulated in prefrontal cortex of App-/- mice ${ }^{40,41}$. We observed increased expression of $J U N$ and FOS mRNAs in APP-KO NPCs (1.3 and 2.7 Log2FC, respectively; Fig. 5A), suggesting that APP also regulate their expression in human context. JUN is activated upon phosphorylation by $\mathrm{JNK}^{42,43,44}$, and we observed a 1.5 -fold increase in phosphoJUN in APP-KO NPCs compared to control (Fig. 5, B and C). Finally, AP1 regulates the expression of BCL6 ${ }^{45}$ in germinal center B cells, and in the developing mouse cortex BCL6, which we found to be upregulated in $A P P-K O$ NPCs, drives neurogenesis of cortical progenitors ${ }^{46}$. Investigating the genes characterizing the $A P P-K O$ specific NP cluster showed enrichment for binding of JUN and JUND by chromatin immunoprecipitation (ChIP-seq) associated with several of these genes (ChEA 2016; Fig. 5, D and E). We also found consensus AP1 binding sites in the regulatory regions of genes upregulated in $A P P-K O$ NPCs, including $D C X$ (Supplementary Table 4). To test whether AP1 activation might drive $A P P-K O$ NPCs into a neurogenic state, we treated APP-KO2 NPC with a specific AP1 inhibitor $(\mathrm{SR} 11302)^{47,48}$ and stained them for SOX2, NEUROG2 and DCX (Fig. 5, F to Q). While the ratio of SOX2+ cells remained unchanged after treatment (Fig. 5R), the number of NEUROG2+ and DCX+ cells was significantly rescued (Fig. 
5, S and T). These results suggest that loss of APP cause premature differentiation through AP1 activation independently of NPC self-renewal.

\section{Loss of APP attenuates the progenitor state of cortical NPCs thorough the canonical WNT} pathway

These data suggest an additional mechanism must be involved in NPC self-renewal downstream of APP. We have recently shown that APP is a receptor for Wnt3a and that Wnt3a binding protects APP from degradation ${ }^{49}$. In addition, our bulk RNAseq data indicated that canonical WNT signaling, which is required in a variety of contexts for stem cell maintenance ${ }^{50}$, is reduced in $A P P$ $K O$ NPCs (fig. S10,C to F), with upregulation of WNT inhibitors $D K K 1, D K K 3, A P C D D 1, A L P K 2$ (Fig. 6A). We compared APP-KO NPCs treated with the canonical ligand WNT3a for 48 hours to untreated control and $A P P-K O$ NPCs and stained them for SOX2 and DCX (Fig. $6 \mathrm{~B}$ to J). We observed a significant rescue of the loss of SOX2+ but no reduction in DCX+ cells (Fig.6, K and L), suggesting that APP regulates the balance between NPC proliferation and differentiation through partially parallel mechanisms.

\section{Discussion}

Here, we present evidence supporting a specific requirement for APP during human cortical development. Loss of APP caused a striking increase in the generation of neurons in a very short time window from human cortical NPCs. Three observations suggest that human brain development may be particularly sensitive to loss of APP. First, there is strongly reduced tolerance to complete of loss of APP in human genetic data ${ }^{14,15},{ }^{16,17}$. Second, both in vivo and in vitro studies on mouse NPCs lacking APP have shown no consistent evidence for altered neurogenesis ${ }^{31-33}$. Third, among the genes whose expression is altered in APP-KO NPCs 33 have been reported to be located within human accelerated regions (HARs), which are conserved genomic loci with elevated divergence in humans ${ }^{51,52}$. We also did not observe an effect on human motor neurogenesis, suggesting the human cortical neurogenesis is particularly sensitive to loss of APP. Because APP is highly conserved in sequence and expression among mammalian species, we speculate that the striking requirement for APP in human cortical neurogenesis is less due to anything special about the human APP protein itself, and more due to the special features of human cortical neurogenesis. In particular, we propose that the links between APP and the Wnt and JNK 
pathways have been co-opted in human, and perhaps more generally primate, cortical NPCs to prolong their ability to retain a progenitor state while also generating more differentiated daughters. Several studies suggest that increased stress drives premature neurogenesis in cortical progenitors $^{53,54}$. APP is a conserved stress response protein, as are JUN and FOS. Therefore, the absence of APP may mimic cellular stress, activate AP1-mediated responses and drive premature neurogenesis. Whether this enhanced requirement for APP in humans may also offer a clue into fAD is worth further investigation. We note that we observed an upregulation of the MAPT gene, encoding the other key AD protein Tau, in APP mutant progenitors (Supplementary Table 3). It is tempting to speculate that fAD mutations in APP have subtle effects on early human cortical development that cause reduced cortical robustness and resistance to a lifetime of neuronal and glial stress eventually contributing to premature neurodegeneration. Finally, although only one case of microcephaly due to a loss of APP has been reported in the medical literature, our findings combined with the low tolerance to the loss of APP in the human population suggest there may be more, and that perhaps the status of APP heterozygosity should be included in clinical genetic counseling.

\section{References and Notes:}

1. Nicolas, M. \& Hassan, B. A. Amyloid precursor protein and neural development. Dev. 141, 2543-2548 (2014).

2. Bergström, P. et al. Amyloid precursor protein expression and processing are differentially regulated during cortical neuron differentiation. Sci. Rep. 6, 1-14 (2016).

3. Coronel, R. et al. Neuronal and Glial Differentiation of Human Neural Stem Cells Is Regulated by Amyloid Precursor Protein (APP) Levels. Mol. Neurobiol. 56, 1248-1261 (2019).

4. Leyssen, M. et al. Amyloid precursor protein promotes post-developmental neurite arborization in the Drosophila brain. EMBO J. 24, 2944-2955 (2005).

5. Hung, C. O. Y. \& Livesey, F. J. Altered $\gamma$-Secretase Processing of APP Disrupts Lysosome and Autophagosome Function in Monogenic Alzheimer's Disease. Cell Rep. 25, 3647-3660.e2 (2018).

6. Kwart, D. et al. A Large Panel of Isogenic APP and PSEN1 Mutant Human iPSC Neurons Reveals Shared Endosomal Abnormalities Mediated by APP $\beta$-CTFs, Not A $\beta$. Neuron 104, 256-270.e5 (2019).

7. Kessissoglou, I. et al. The Drosophila Amyloid Precursor Protein homologue mediates neuronal survival and neuro-glial interactions. (2020) doi:10.1101/2020.03.09.983353.

8. Sayer, R., Robertson, D., Balfour, D. J. K., Breen, K. C. \& Stewart, C. A. The effect of stress on the expression of the amyloid precursor protein in rat brain. Neurosci. Lett. 431, (2008).

9. Salminen, A., Kauppinen, A. \& Kaarniranta, K. Hypoxia/ischemia activate processing of Amyloid Precursor Protein: impact of vascular dysfunction in the pathogenesis of 
Alzheimer's disease. Journal of Neurochemistry vol. 140 (2017).

10. Chen, G. F. et al. Amyloid beta: Structure, biology and structure-based therapeutic development. Acta Pharmacol. Sin. 38, 1205-1235 (2017).

11. Coronel, R. et al. Physiological effects of amyloid precursor protein and its derivatives on neural stem cell biology and signaling pathways involved. Neural Regen. Res. 14, 1661 (2019).

12. Müller, U. C., Deller, T. \& Korte, M. Not just amyloid: Physiological functions of the amyloid precursor protein family. Nat. Rev. Neurosci. 18, 281-298 (2017).

13. O'Brien, R. J. \& Wong, P. C. Amyloid precursor protein processing and alzheimer's

14. Lek, M. et al. Analysis of protein-coding genetic variation in 60,706 humans. Nature 536, (2016).

15. Karczewski, K. J. et al. The mutational constraint spectrum quantified from variation in 141,456 humans. Nature 581, (2020).

16. Klein, S. et al. Truncating mutations in APP cause a distinct neurological phenotype. Ann. Neurol. 80, 456-460 (2016).

17. Nguyen, K. V., Leydiker, K., Wang, R., Abdenur, J. \& Nyhan, W. L. A neurodevelopmental disorder with a nonsense mutation in the Ox-2 antigen domain of the amyloid precursor protein (APP) gene. Nucleosides, Nucleotides and Nucleic Acids 36, 317-327 (2017).

18. Lorent, K. et al. Expression in mouse embryos and in adult mouse brain of three members of the amyloid precursor protein family, of the alpha-2-macroglobulin receptor/low density lipoprotein receptor-related protein and of its ligands apolipoprotein E, lipoprotein lipase, . Neuroscience 65, 1009-1025 (1995).

19. Young-Pearse, T. L. et al. A critical function for $\beta$-amyloid precursor protein in neuronal migration revealed by in utero RNA interference. J. Neurosci. 27, 14459-14469 (2007).

20. Polioudakis, D. et al. A Single-Cell Transcriptomic Atlas of Human Neocortical Development during Mid-gestation. Neuron 103, 785-801.e8 (2019).

21. Small, D. H. et al. $\beta$-Amyloid precursor protein: Function in stem cell development and alzheimer's disease brain. Neurodegener. Dis. 13, 96-98 (2014).

22. Coronel, R. et al. Role of Amyloid Precursor Protein (APP) and Its Derivatives in the Biology and Cell Fate Specification of Neural Stem Cells. Mol. Neurobiol. 55, 7107-7117 (2018).

23. Wang, S. et al. Amyloid $\beta$ precursor protein regulates neuron survival and maturation in the adult mouse brain. Mol. Cell. Neurosci. 77, 21-33 (2016).

24. Caillé, I. et al. Soluble form of amyloid precursor protein regulates proliferation of progenitors in the adult subventricular zone. Development 131, 2173-2181 (2004).

25. Otani, T., Marchetto, M. C., Gage, F. H., Simons, B. D. \& Livesey, F. J. 2D and 3D Stem Cell Models of Primate Cortical Development Identify Species-Specific Differences in Progenitor Behavior Contributing to Brain Size. Cell Stem Cell 18, 467-480 (2016).

26. Suzuki, I. K. et al. Human-Specific NOTCH2NL Genes Expand Cortical Neurogenesis through Delta/Notch Regulation. Cell 173, 1370-1384.e16 (2018).

27. Genin, E. C. et al. Mitochondrial defect in muscle precedes neuromuscular junction degeneration and motor neuron death in CHCHD10 S59L/+ mouse. Acta Neuropathol. 138, 123-145 (2019).

28. Molyneaux, B. J., Arlotta, P., Menezes, J. R. L. \& Macklis, J. D. Neuronal subtype 
specification in the cerebral cortex. Nat. Rev. Neurosci. 8, 427-437 (2007).

29. Gaspard, N. et al. An intrinsic mechanism of corticogenesis from embryonic stem cells. Nature 455, 351-357 (2008).

30. Nowakowski, T. J. et al. Spatiotemporal gene expression trajectories reveal developmental hierarchies of the human cortex. Science (80-. ). 358, 1318-1323 (2017).

31. Ma, Q. H. et al. A TAG1-APP signalling pathway through Fe65 negatively modulates neurogenesis. Nat. Cell Biol. 10, 283-294 (2008).

32. $\mathrm{Hu}, \mathrm{Y}$. et al. Role of cystatin $\mathrm{C}$ in amyloid precursor protein-induced proliferation of neural stem/progenitor cells. J. Biol. Chem. 288, (2013).

33. Bergmans, B. A. et al. Neurons generated from APP/APLP1/APLP2 triple knockout embryonic stem cells behave normally in vitro and in vivo: Lack of evidence for a cell autonomous role of the amyloid precursor protein in neuronal differentiation. Stem Cells 28, 399-406 (2010).

34. Johnson, M. B. et al. Single-cell analysis reveals transcriptional heterogeneity of neural progenitors in human cortex. Nat. Neurosci. 18, (2015).

35. Urbán, N. \& Guillemot, F. Neurogenesis in the embryonic and adult brain: Same regulators, different roles. Frontiers in Cellular Neuroscience vol. 8 (2014).

36. Liu, C. et al. APP upregulation contributes to retinal ganglion cell degeneration via JNK3. Cell Death Differ. 25, (2018).

37. Kögel, D., Schomburg, R., Copanaki, E. \& Prehn, J. H. M. Regulation of gene expression by the amyloid precursor protein: Inhibition of the JNK/c-Jun pathway. Cell Death Differ. 12, (2005).

38. Copanaki, E. et al. SAPP $\alpha$ antagonizes dendritic degeneration and neuron death triggered by proteasomal stress. Mol. Cell. Neurosci. 44, 386-393 (2010).

39. Eckert, G. P. et al. Liposome-incorporated DHA increases neuronal survival by enhancing non-amyloidogenic APP processing. Biochim. Biophys. Acta-Biomembr. 1808, 236-243 (2011).

40. Hendrickx, A. et al. Epigenetic Induction of EGR-1 Expression by the Amyloid Precursor Protein during Exposure to Novelty. PLoS One 8, (2013).

41. Hendrickx, A. et al. Epigenetic regulations of immediate early genes expression involved in memory formation by the amyloid precursor protein of alzheimer disease. PLoS One 9 , (2014).

42. Kallunki, T., Deng, T., Hibi, M. \& Karin, M. c-Jun can recruit JNK to phosphorylate dimerization partners via specific docking interactions. Cell 87, 929-939 (1996).

43. Barilá, D. et al. A nuclear tyrosine phosphorylation circuit: c-Jun as an activator and substrate of c-Abl and JNK. EMBO J. 19, 273-281 (2000).

44. Pulverer, B. J., Kyriakis, J. M., Avruch, J., Nikolakaki, E. \& Woodgett, J. R. Phosphorylation of c-jun mediated by MAP kinases. Nature 353, 670-674 (1991).

45. Arguni, E. et al. JunD/AP-1 and STAT3 are the major enhancer molecules for high Bcl6 expression in germinal center B cells. Int. Immunol. 18, 1079-1089 (2006).

46. Tiberi, L. et al. BCL6 controls neurogenesis through Sirt1-dependent epigenetic repression of selective Notch targets. Nat. Neurosci. 15, (2012).

47. Keasey, M. P., Kang, S. S., Lovins, C. \& Hagg, T. Inhibition of a novel specific neuroglial integrin signaling pathway increases STAT3-mediated CNTF expression. Cell Commun. Signal. 11, (2013).

48. Mishra, D. K. \& Kim, M. P. SR 11302, an AP-1 Inhibitor, Reduces Metastatic Lesion 
Formation in Ex Vivo 4D Lung Cancer Model. Cancer Microenviron. 10, (2017).

49. Liu, T., Nicolas, M., Zhang, T., Rice, H., Soldano, S., Claeys, S., Petrova, I.M., Fradkin, L.G., De Strooper, B., and \& Hassan, B.A. The Amyloid Precursor Protein is a conserved Wnt receptor. bioRxiv 2021.01.18.426557; doi:https://doi.org/10.1101/2021.01.18.426557 (2021).

50. Mutch, C. A., Schulte, J. D., Olson, E. \& Chenn, A. Beta-catenin signaling negatively regulates intermediate progenitor population numbers in the developing cortex. PLoS One 5, (2010).

51. Levchenko, A., Kanapin, A., Samsonova, A. \& Gainetdinov, R. R. Human accelerated regions and other human-specific sequence variations in the context of evolution and their relevance for brain development. Genome Biology and Evolution vol. 10 (2018).

52. Doan, R. N. et al. Mutations in Human Accelerated Regions Disrupt Cognition and Social Behavior. Cell 167, 341-354.e12 (2016).

53. $\mathrm{Hu}, \mathrm{Q}$. et al. Oxidative stress promotes exit from the stem cell state and spontaneous neuronal differentiation. Oncotarget 9, 4223-4238 (2018).

54. Pérez Estrada, C., Covacu, R., Sankavaram, S. R., Svensson, M. \& Brundin, L. Oxidative stress increases neurogenesis and oligodendrogenesis in adult neural progenitor cells. Stem Cells Dev. 23, 2311-2327 (2014).

Acknowledgments: This work was supported by the Investissements d'Avenir program (ANR10-IAIHU-06), Paris Brain Institute-ICM core funding and the Roger De Spoelberch Foundation Prize (to B.A.H.). Tengyuan Liu is funded by the Chinese Scholarship Council (CSC). We thank Natalia Baumann and Dr. Denis Jabaudon for sharing the pseudotime analysis code, Dr. Bart De Strooper for sharing the anti-APP antibody, Drs. Stephane Nedelec, Joris De Wit, Denis Jabaudon, members of the Hasan lab as well as Antoine Graindorge, and Stuart Edelstein from Scipio biosciences for helpful discussions. Scipio bioscience was supported by the Investissements d'Avenir program and the Région Île-de-France. This work benefited from equipment and services from the ICM's genotyping and sequencing core facility (iGenSeq). Mouse breeding work was conducted at the PHENO-ICMice facility. The Core is supported by 2 Investissements d'Avenir grants (ANR-10- IAIHU-06 and ANR-11-INBS-0011-NeurATRIS) and the "Fondation pour la Recherche Médicale". iPSC work was carried out at the CELIS core facility with support from Program Investissements d'Avenir (ANR-10-IAIHU-06). Light microscopy was carried out at the ICM.Quant facility. We thank all core technical staff involved and in particular Stephanie Bigou from CELIS for her advice on iPSCs and CRISPR/Cas9 work and Claire Lovo from ICM.Quant for help with image analysis.

Materials and Methods

iPSC culture and maintenance

iPSC cell lines WTS002 (iPSC line1) and WTS008 (iPSC line2) were purchased from EBISC (European bank for induced pluripotent cells). They were maintained on Geltrex LDEV-Free hESC-qualified Reduced Growth Factor Basement Membrane Matrix (ThermoFisher Scientific) in Essential $8^{\mathrm{TM}}$ Flex Media Kit (ThermoFisher Scientific) with $0.1 \%$ penicillin/streptomycin. Cultures were fed every other day and passaged every 5-7 days by ReLeSR ${ }^{\mathrm{TM}}$ (STEMCELL Technologies).

\section{Creating $A P P-K O$ clones by CRISPR/Cas9}




\section{iPSC line1}

Four guide RNAs were designed by Crispor (http://crispor.tefor.net/) to target the APP first exon and cloned in the vector pCAG-CAS9-GFP (Extended data Fig. 1a, b). To evaluate the cleavage efficiencies of the guide RNAs, HEK293 cells were transfected by expression vector p-CAG-Cas9-GFP containing four guide RNAs (Extended data Fig. 1c). DNA extraction and PCR were performed 48 hours post transfection for the cleaved region. Sequenced PCR products were analyzed in TIDE (Tracking of Indels by Decomposition) to obtain the cleavage efficiency. The best guide RNA with $8.5 \%$ total efficiency and lowest off-target was chosen for further experiments (Extended data Fig. 1d). The iPSC line was transfected by the expression vector containing the best guide RNA using Lipofectamin stem reagent (ThermoFisher Scientific). GFP positive cells isolated by FACS 48 hours post-transfection and seeded at low density. A total of 118 clones were picked after 5 days, expanded, and 60 screened for possible mutation (Extended data Fig. 1e-h), and 16 out of the 60 clones had homozygous mutations for APP. These mutations are classified as: 1, 2, 3, 20, 22, 33bp deletions and $1 \mathrm{bp}$ and $2 \mathrm{bp}$ insertions (Supplementary Table 5). The absence of expression of the APP protein was confirmed by western blot in 14 clones (Extended data Fig. 1i). The efficiency of producing APP knock-out clones was 23\% (Figure 1j). Three APP knock out clones were chosen for further experiments and low APP mRNA levels were confirmed by quantitative-PCR (Extended data Fig. 1k). The control, which was chosen for further experiments, expressed guide RNA and CAS9 but the APP gene remained un-mutated. The pluripotency of $A P P-K O$ cells and the control were confirmed by OCT4 staining (Extended data Fig. 2 d-g).

\section{iPSC line2}

The guide RNA 1 and the ribonucleoprotein approach were used to generate $A P P-K O .1 \times 10^{6} \mathrm{iPSCs}$ were nucleofected with the RNP complex from IDT (225pmol of each RNA and 120pmol of Cas9 protein). The iPSCs were plated 48 hrs later at very low density $\left(10\right.$ cells $\left./ \mathrm{cm}^{2}\right)$ on Laminin-521 with CloneR supplement (Stem Cell Technology) for clonal selection. One week later, iPSC clones were picked under a stereomicroscope and cultured on Laminin-521 in 96 well plates (Duscher). The resulting iPSC clones were duplicated after confluency and used for cryoconservation and DNA extraction. One base-pair deletion was observed in screening results and the absence of expression of APP protein was confirmed by western blot. The APP knock out clone and control were used for further experiments and the low level of APP mRNA was confirmed by quantitative-PCR (Extended data Fig. 11, m, n).

\section{Cortical differentiation of human iPSC}

Three $A P P-K O$ and one $A P P-W T$ control clones underwent a cortical differentiation protocol to produce cortical neurons in 2D. The protocol is divided into 2 main stages: neural induction and neural differentiation. At day 0, iPSCs were detached by Accutase (ThermoFisher Scientific) and transferred to T75 ultra-low attachment flasks (VWR) in Essential 8 medium with $0.1 \%$ penicillin/streptomycin and Stemgent hES Cell Cloning \& Recovery Supplement (Ozyme, 01-0014-500) to form Embryoid body (EB). At day 1, the medium was switched to EB medium contain DMEM/F12 Glutamax, 20\% knockout serum replacement, 1\% nonessential amino acids, $1 \%$ penicillin/streptomycin,

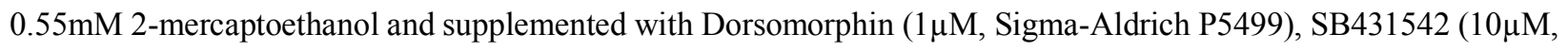
Abcam ab120163) for 8 days. At day 8, Embryoid bodies (EBs) were collected and seeded on Geltrex and maintained for 6-8 days in Neurobasal without vitamin A medium and B27 without vitamin A supplemented with Human 
EGF(10ng/ml) and human FGF2 (10ng/ml). Cells were fed every day from day 0 to day 16 . Rosettes were manually picked and dissociated with Accutase, then seeded on poly-ornithine and laminin-coated dishes for expansion. They were maintained with passage for two additional weeks to achieve a large pool of neural precursor cells (NPCs) (Extended data Fig. 2 a-c). Characterization for NPCs was performed by staining against Nestin, SOX2, SOX1, and PAX6 (Extended data Fig. 2 h1-k6). NPCs were seeded on 24-well plates with coverslips and the day after seeding, the medium was switched to the B-27 Plus Neuronal Culture System (ThermoFisher Scientific) supplemented with Ascorbic acid. Cortical neurons were stained for early-born neural markers and late-born neural markers at different time point. They were kept for 3 to 4 months in culture and stained for synaptic markers at 60 days and 120 days post differentiation.

\section{Motor neuron differentiation and staining}

Control and mutant iPSC clones were differentiated into motor neurons as described ${ }^{1}$. In summary, after dissociation of EBs containing motor neuron progenitors at D10, single cells were plated on poly ornithine-laminin coated coverslips at 2x10e5 cells/coverslip for 1, 4 and 7 more days. Cells were fixed with 4\% paraformaldehyde in PBS for 10 minutes RT. For staining, cells were treated with $5 \%$ goat serum with $0.1 \%$ Triton X100 (Thermofisher Scientific) for 1 hour at RT, and then incubated overnight at $4{ }^{\circ} \mathrm{C}$ with a mouse anti- $\beta$ III-Tubulin (TUJ1, Sigma, 1/500) and a rabbit anti-ISLET1 (Abcam, 1/100) antibodies in 5\% goat serum. Secondary antibodies (goat anti-mouse IgG2a-Alexa 555 (1/2000) and goat anti-rabbit IgG Alexa 488 (1/2000) from ThermoFischer Scientific) were incubated one hour at RT with Hoechst33342 to stain nuclei.

\section{Neural differentiation follow-up by viral vector}

Neural differentiation follow-up was performed by the viral vector approach described in previous studies ${ }^{2,3} . A P P$ $K O$ and control neural precursor cells were seeded on poly ornithine-laminin coated coverslips and transduced by virus Lenti-Synapsin-GFP. The medium was changed to fresh Neurobasal without vitamin A and B27 without vitamin A supplemented with EGF and hFGF2 to wash out lentiviruses one day after initial infection. When the GFP+ cells appeared, it was considered as day 0 and the medium was switched to differentiation medium (B-27 Plus Neuronal Culture System) and kept for 7 and 30 days. Lentiviral expressing cells were detected by anti-GFP antibody and cell type was determined by anti-SOX2 and anti-DCX antibodies.

\section{Immunocytochemistry}

Cell were fixed in PFA 4\% and incubated for $30 \mathrm{~min}$ in blocking buffer (phosphate-buffered saline and Triton X-100 $0.3 \%$ and $5 \%$ horse serum). Primary antibodies (Supplementary Table 6) were diluted in antibody solution (phosphatebuffered saline and Triton X-100 0.3\% and 5\% horse serum) and applied overnight at $4{ }^{\circ} \mathrm{C}$. After three washes in phosphate-buffered saline, secondary antibodies conjugated to Alexa fluorophores (Molecular Probes, Eugene, OR, USA) were diluted at 1:1000 in blocking buffer and applied for $1 \mathrm{hr}$ at room temperature. Cells were washed in phosphate-buffered saline followed by nuclear staining by DAPI (Sigma) for $10 \mathrm{~min}$. Cells was washed three more 
times and mounted by VECTASHIELD® Mounting Medium-Vector Laboratories. Confocal image acquisition was performed using Olympus FV1200 and SP8 Leica DLS.

\section{Generation of cortical organoids and immunohistochemistry}

Cerebral organoids were generated from human iPSCs using a previously reported protocol ${ }^{4}$ with some modifications. The hiPSCs were incubated with Accutase (Life Technologies, A1110501) at $37{ }^{\circ} \mathrm{C}$ for 7 min and dissociated into single cells. In order to obtain uniformly sized spheroids, approximately $3 \times 10^{6}$ single cells were added per well in the AggreWell 800 plate (STEMCELL Technologies, 34815) with Essential 8 flex medium supplemented with Stemgent hES Cell Cloning \& Recovery Supplement (1X, Ozyme, STE01-0014-500) and incubated at 37 ${ }^{\circ}$ with 5\% CO2. After $24 \mathrm{hr}$, spheroids from each microwell were collected by firmly pipetting medium (with a cut end of a P1000 tip) in the well up and down and transferring it into Corning® non-treated culture dishes (Merck, CLS430591500EA) in TeSR ${ }^{\text {TM}}$-E6 (StemCell Technologies, \#05946) supplemented with two inhibitors of the SMAD signalling

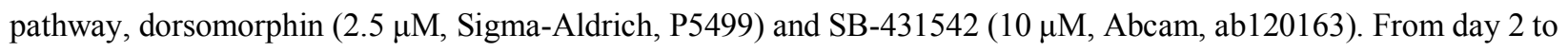
day 5, TeSR TM-E6 supplemented with dorsomorphin and SB-431542 was changed every day. On the sixth day in suspension, neural spheroids were transferred to neural medium containing Neurobasal minus vitamin A (Life Technologies, 10888), B-27 supplement without vitamin A (Life Technologies, 12587), GlutaMax (1\%, Life Technologies, 35050), Penicillin-Streptomycin (1\%, Life technologies, 15140122) and 2-mercaptoethanol (5mM, Life technologies, 31350010). The neural medium was supplemented with $10 \mathrm{ng} / \mathrm{mL}$ epidermal growth factor (PreproTech, AF-100-15) and $10 \mathrm{ng} / \mathrm{mL}$ basic fibroblast growth factor (R\&D Systems, 234-FSE-025) for $19 \mathrm{~d}$ (until day 24), with medium changed daily during the first 10 days and every other day for the subsequent 9 days. At day 15, spheroids were fixed with $4 \%$ paraformaldehyde in PBS overnight at $4^{\circ} \mathrm{C}$. Following 2 washings with PBS $+2 \%$ Triton-X100 for $2 \mathrm{hr}$, spheroids were treated overnight at RT with PBS $+2 \%$ Triton-X100 + 2\%Tween20 + 20\%DMSO. Blocking/permeabilization was performed with PBS $+10 \%$ Horse serum , 3\% BSA, and 2\% Triton-X100 for $24 \mathrm{hr}$ at RT. Primary antibodies, (Sox2 1/500, Milipore AB5603) and (DCX 1/2000, Milipore AB2253), were incubated in the same solution supplemented with $0.05 \%$ Azide for at least 3 days at $4{ }^{\circ} \mathrm{C}$. After multiple washing with PBS $+0.5 \%$ Tween-20 until the next day, spheroids were then incubated for at least 3 days with secondary antibodies. After 2 days of washing with PBS $+0.5 \%$ Tween-20, samples were cleared overnight in RapiClear 1.49 (SunJin lab) before mounting on cavity slides. Sample were imaged using confocal microscope Nikon A1R HD25 with a 10X objective (MRD71120).

\section{Embryo collection, immunohistochemistry, and antibodies}

E10.5 embryos were collected from APP-wild type or APP-knockout pregnant mice, whole embryos were fixed in $2 \%$ paraformaldehyde (PFA) in PBS at $4{ }^{\circ} \mathrm{C}$ for $2-3$ hours, then dehydrated in $30 \%$ sucrose in $1 \mathrm{X}$ PBS overnight (o/n). After all the samples sank into the bottom of the tube, they were embedded in OCT compound (TissueTek) and frozen at $-20{ }^{\circ} \mathrm{C}$. Sagittal sections were performed by cryostat (Leica) at $20 \mu \mathrm{m}$ and then slices stored at $-80{ }^{\circ} \mathrm{C}$. For the immunostaining, sections were fixed with 4\% PFA for 10 minutes at RT, then blocked with $10 \%$ normal donkey or goat serum in $1 \mathrm{X}$ PBS with $0.1 \%$ Triton (PBT) for 1 hour at RT followed by 3 washes in $1 \mathrm{X}$ PBT. Thereafter, these 
sections were incubated with primary antibodies diluted in $0.1 \% 1 \mathrm{X}$ PBT containing $1 \%$ normal donkey or goat serum $\mathrm{o} / \mathrm{n}$ at $4{ }^{\circ} \mathrm{C}$ or 3-4 hours at RT. After 3 washes with $1 \mathrm{X}$ PBT, samples were incubated with appropriate secondary antibodies conjugated with Alexa Fluor 488, Alexa Fluor 555, or Alexa Fluor 647 (1:500, Invitrogen) in 0.1\% 1 X PBT containing 1\% normal donkey or goat serum for 1-2 hours at RT. After washing with 1X PBT for 3 times, then counterstained with DAPI (1:2000, Sigma), the slides were mounted by using Vectashield (Vector) after rinsing. Primary antibodies used in this study were mouse anti-TUJ1 (1:300, Biolegend: 801202), rabbit anti-Pax6 (1:300, Biolegend: PRB-278P). After staining, images were obtained by using confocal microscope Olympus FV-1200.

\section{qPCR}

The cells were lysed directly in the wells by addition of $300 \mu$ Buffer RLT supplemented with $15 \mathrm{mM}$ betamercaptoethanol (Thermo Fisher Scientific) after a wash with Dulbecco's Phosphate-Buffered Saline (DPBS, Life Technologies). Total RNAs were isolated using the RNeasy Mini extraction kit (Qiagen, Courtaboeuf, France) according to the manufacturer's protocol. RNA levels and quality were quantified using a Nanodrop spectrophotometer. cDNA synthesis was performed by Thermo Scientific Verso cDNA Synthesis Kit and Quantitative PCR assay was performed by Sybergreen Gene Expression Assays in triplicate wells of 96-well plates. Primers are listed in supplementary Table 7 and $2^{-(\mathrm{Cp} \text { GOI - Cp internal gene) }}$ was used for analysis. Housekeeping gene (GAPDH) was selected to control variation in cDNA amounts.

\section{Bulk RNA sequencing and analysis}

The cells were lysed directly in the wells by addition of $300 \mu$ Buffer RLT supplemented with $15 \mathrm{mM}$ betamercaptoethanol (Thermo Fisher Scientific) after a wash with Dulbecco's Phosphate-Buffered Saline (DPBS, Life Technologies). Total RNA was isolated using the RNeasy Mini extraction kit (Qiagen, Courtaboeuf, France) according to the manufacturer's protocol. RNA levels and quality were quantified using a Nanodrop spectrophotometer. RNA sample purity/integrity was assessed using an Agilent 2200 Tapestation. mRNA library preparation was completed following manufacturer's recommendations (KAPA mRNA hyperprep ROCHE). Final samples pooled library prep were sequenced on Nextseq 500 ILLUMINA with MidOutPut cartridge (2x130Million 75 base reads) with 1 run, corresponding to $2 \times 20$ Million reads per sample after demultiplexing. The quality of raw data was evaluated with

FastQC. Poor quality sequences were trimmed or removed with fastp software to retain only good quality paired reads without adapters. Star v2.5.3 $\mathrm{a}^{5}$ was used to align reads on the hg19 reference genome using standard options. Quantification of gene and isoform abundances was carried out with rsem 1.2.28 , prior to normalisation on library size with the edge $\mathrm{R}^{7}$ bioconductor package. Finally, differential analysis was conducted with the glm framework likelihood ratio test from edgeR. Multiple hypothesis adjusted p-values were calculated with the Benjamini-Hochberg ${ }^{8}$ procedure to control FDR.

\section{Single cell RNA sequencing and analysis}

For each control and knockout cell lines, single cell samples were prepared for 3' mRNA sequence determinations

using the Scipio bioscience protocol (to be published) with barcoding beads (NxSeq Single-cell RNA-seq Beads, LGC Biosearch Technologies). For beads that captured mRNA molecules, reverse transcription, PCR amplification of cDNA, and sequencing library preparation were performed according to published procedures ${ }^{9}$. A total of $35,106,546$ 
reads, 56,108,512 reads, and 54,702,467 reads were generated by NovaSeq from two replicates of $A P P-K O 2$ (KO2-1 and KO2-2) and one CT (control) sample, corresponding to 2000, 2000, and 1500 cells. The reads passed QC process using FASTQC v0.11.8. Sample analyses were performed using UMI-tools v1.0.0. Reads were aligned to GRCh38.94 using STAR v2.7 with default parameters. FeatureCounts v1.6.4 (Ensembl GRCh38.94.GTF) was used to count the number of aligned reads per feature, followed by umi_tools dedup to collapse those reads belonging to a barcode and mapping the same position of a gene. To build the count table umi_tools count was applied. Seurat V3.1.4 ${ }^{10}$ was used: i) to filter cells having more than 200 genes and less than $20 \%$ mitochondrial genes, with 1326 , 1594, and 1299 cells passing the QC filters for the KO2-1, KO2-2, and CT samples, respectively.; ii) to pool both KO samples (2920 total); iii) to cluster $\mathrm{KO}$ and $\mathrm{CT}$ cells using Seurat clustering with 10 PCA dimensions and resolution 0.5; iv) to identify cluster markers using FindAllMarkers with default parameters. Visualizations were performed with Seurat and ggplot2 packages. DEGs in KO vs CT were analyzed for enrichment in gene ontology, biological and disease associations using Enrichr tools to further explore ChIP-seq datasets linking transcription regulators with DEGs ${ }^{11}$.

\section{Rescue experiment}

Neural precursor cells of Control and $A P P-K O 2$ were transfected with $p P B-C A G-I R E S-E G F P$ and $A P P-K O 2$ NPC were transfected by $p P B-C A G-h A P P-I R E S-E G F P$ and kept for 4 days. Cells were fixed and stained for GFP/SOX2/DCX. The GFP+ cells were quantified, and cell type was determined by SOX2 and DCX as progenitor and differentiated cell, respectively.

\section{Neural precursor treatment}

WNT3a (R\&D Systems) and SR11302 (TOCRIS) were used at final concentrations of $150 \mathrm{ng} / \mathrm{ml} \mathrm{and} 10 \mu \mathrm{M}$, respectively. Neural precursor cells of control and $A P P-K O 2$ were treated for 48 hours and then stained for SOX2 and DCX.

\section{Western blot}

Cells were place on ice, lysed directly in wells by adding RIPA buffer (Sigma) supplemented with Complete protease inhibitor cocktail (Roche) and agitated for $20 \mathrm{~min}$. Thereafter, the samples were collected and centrifuged at $>14000$ g, 30min at $4{ }^{\circ} \mathrm{C}$ and the supernatant was transferred to a fresh tube. Protein determination was performed using the Pierce $^{\mathrm{TM}}$ Detergent Compatible Bradford Assay Kit (ThermoFisher Scientific). Sample buffer (BOLT LDS, Life technologies) was added to equal amounts of protein, and samples were loaded onto a BOLT 4-12\% Bis-tris gel and transferred using MES buffer (all from Life technologies). Proteins were blotted onto a $0.2 \mu \mathrm{M}$ nitrocellulose membrane (GE Healthcare) using semi-dry technique. Membranes were blocked in 5\% Nonfat-Dried Milk bovine (Sigma) and incubated over night at $4{ }^{\circ} \mathrm{C}$ with primary antibody. After washing, membranes were incubated with HRP-conjugated secondary antibodies (supplemented table) for 1 hour at room temperature. Protein detection was performed by Pierce ECL Western Blotting substrate (ThermoFisher Scientific) and bands were visualized using ChemiDoc $^{\mathrm{TM}}$ Touch Imaging System (BioRad laboratories). Band intensities were calculated using Image Lab software. 


\section{Statistical analysis}

Statistical analysis was performed by GraphPad Prism. Data in figure panels reflect 3 independent experiments performed on different days. An estimate of variation within each group of data is indicated using standard error of the mean (SEM). We performed unpaired $t$ test for assessing the significance of differences in the analyses containing two conditions, one-way ANOVA correction in the analyses containing more than three conditions and two-way ANOVA in the group analysis.

\section{References:}

1. Genin, E. C. et al. Mitochondrial defect in muscle precedes neuromuscular junction degeneration and motor neuron death in CHCHD10 S59L/+ mouse. Acta Neuropathol. 138, 123-145 (2019).

2. Otani, T., Marchetto, M. C., Gage, F. H., Simons, B. D. \& Livesey, F. J. 2D and 3D Stem Cell Models of Primate Cortical Development Identify Species-Specific Differences in Progenitor Behavior Contributing to Brain Size. Cell Stem Cell 18, 467-480 (2016).

3. Suzuki, I. K. et al. Human-Specific NOTCH2NL Genes Expand Cortical Neurogenesis through Delta/Notch Regulation. Cell 173, 1370-1384.e16 (2018).

4. Sloan, S. A., Andersen, J., Pașca, A. M., Birey, F. \& Pașca, S. P. Generation and assembly of human brain region-specific three-dimensional cultures. Nat. Protoc. 13, (2018).

5. Dobin, A. et al. STAR: Ultrafast universal RNA-seq aligner. Bioinformatics 29, 15-21 (2013).

6. Li, B. \& Dewey, C. N. RSEM: Accurate transcript quantification from RNA-seq data with or without a reference genome. Bioinforma. Impact Accurate Quantif. Proteomic Genet. Anal. Res. 41-74 (2014) doi:10.1201/b16589.

7. Robinson, M. D., McCarthy, D. J. \& Smyth, G. K. edgeR: A Bioconductor package for differential expression analysis of digital gene expression data. Bioinformatics 26, 139-140 (2009).

8. Benjamini, Y. \& Hochberg, Y. Controlling the False Discovery Rate: A Practical and Powerful Approach to Multiple Testing. J. R. Stat. Soc. Ser. B 57, 289-300 (1995).

9. Macosko, E. Z. et al. Highly parallel genome-wide expression profiling of individual cells using nanoliter droplets. Cell 161, (2015).

10. Stuart, T. et al. Comprehensive Integration of Single-Cell Data. Cell 177, (2019).

11. Chen, E. Y. et al. Enrichr: Interactive and collaborative HTML5 gene list enrichment analysis tool. BMC Bioinformatics 14, (2013). 


\section{Shabani-Fig.1}
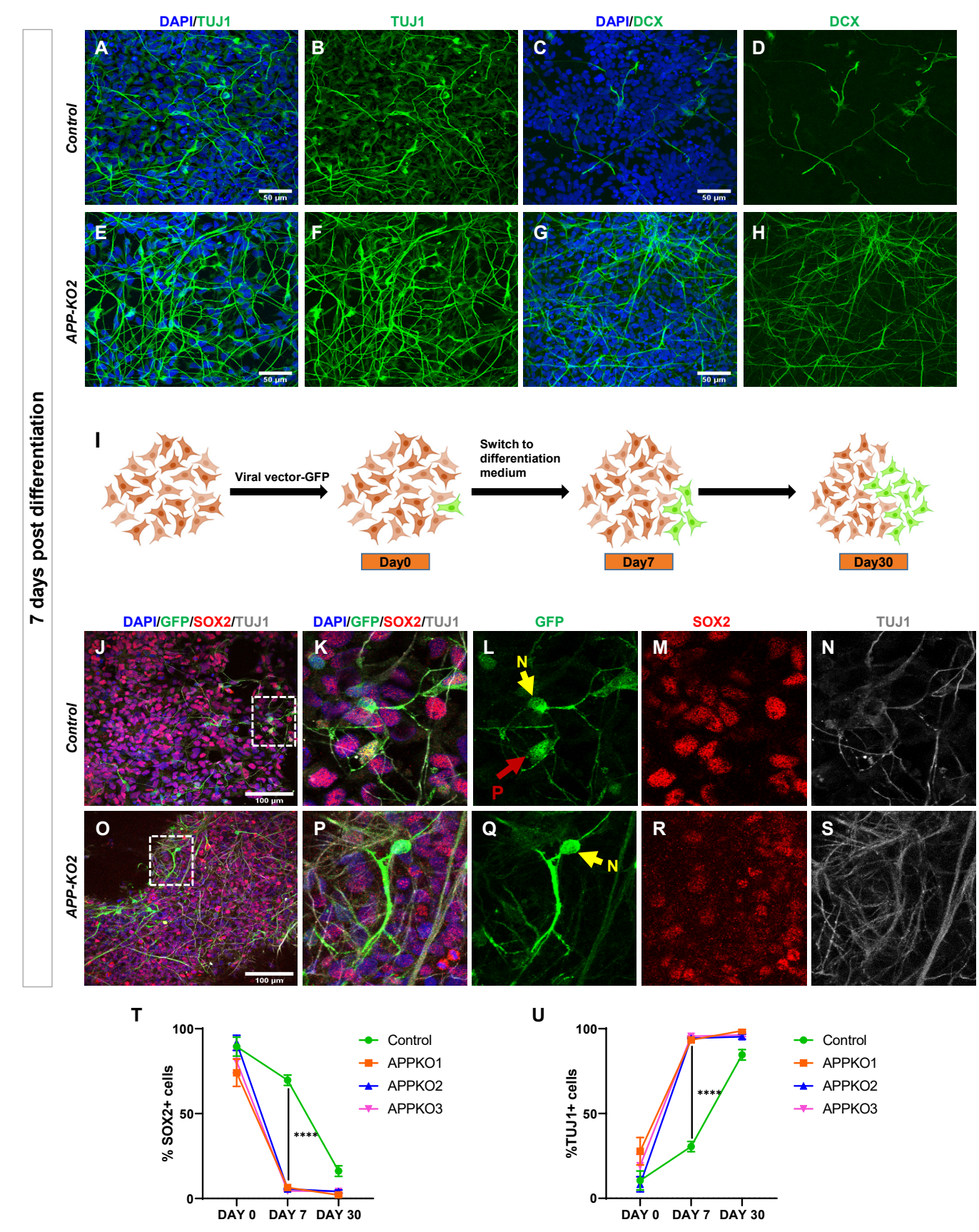

Fig. 1. Accelerated neurogenesis from $A P P-K O 2$ NPCs. a-h, Expression of the neuronal markers TUJ1 and DCX in APP-KO2 compared to control (Scale bar $50 \mu \mathrm{m})$. i, Schematic illustration of sparse labelling using a lentiviral vector approach to follow neurogenesis. $\mathbf{j}-\mathbf{s}$, Expression of SOX2 and TUJ1 within GFP+ cells in control and APP-KO2 7 days post differentiation (Scale bar 100 $\mu \mathrm{m}, \mathrm{N}=$ neuron, $\mathrm{P}=$ Progenitor). $\mathbf{t}$, Increase in TUJ1+ and $\mathbf{u}$, Decrease in SOX2 expression in $A P P$ $K O s$ neurons compared to control at 7 days (2way ANOVA, $\mathrm{p}<0.0001$ ), but not at 30 days post differentiation. 


\section{Shabani-Fig.2}

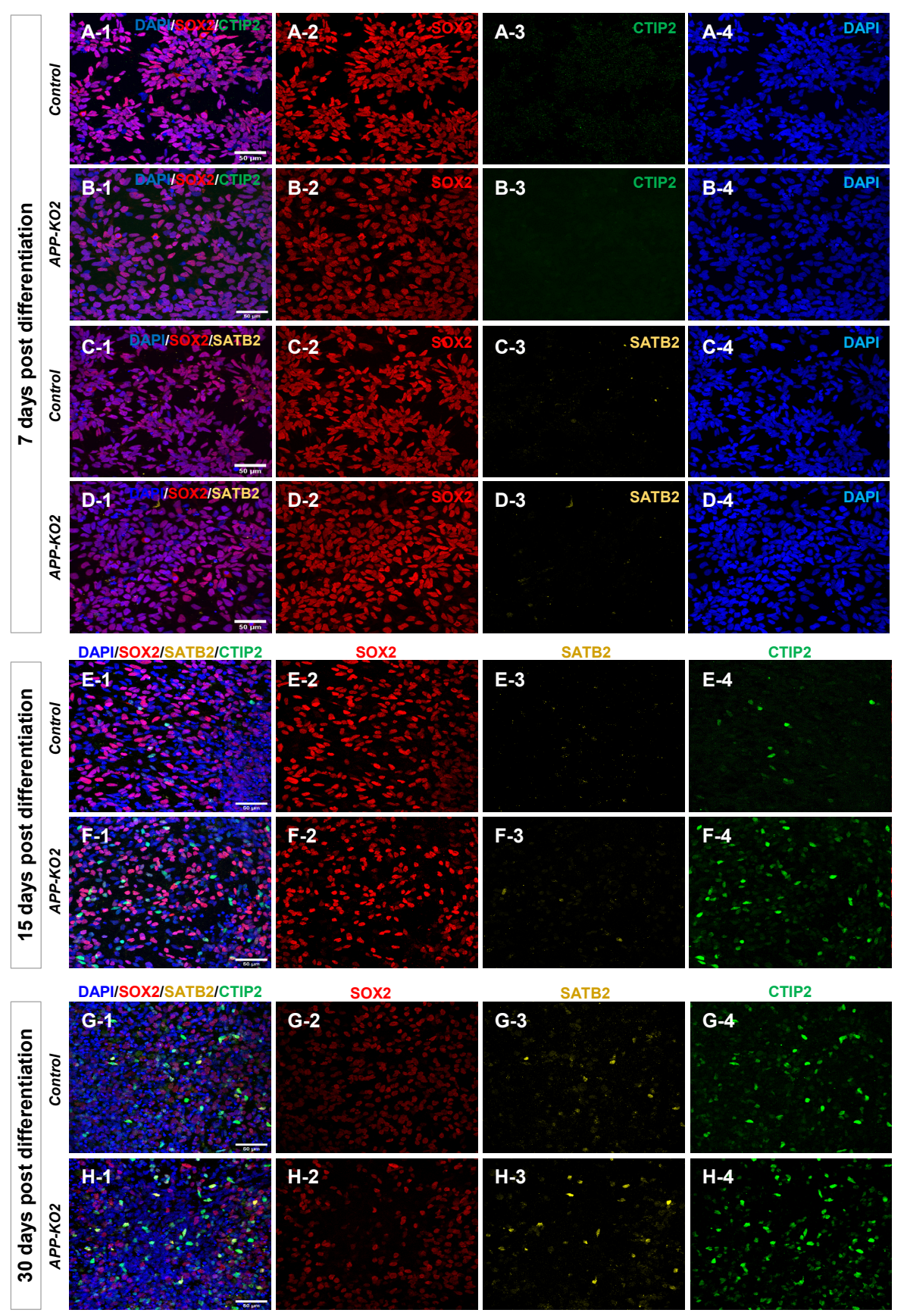

Fig. 2. Cortical neuron fate markers appear with same temporal order in $A P P-K O$ and controls. a1-d4, No expression of CTIP2 (early born neuron) and SATB2 (late born neuron) was observed 7 days post differentiation. e1-f4, CTIP2 and SATB2 appeared at the same time 15 days post differentiation in control and $A P P-K O 2$ (scale bar $50 \mu \mathrm{m}$ ). g1-h4, Expression of CTIP2 and

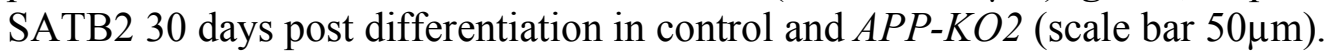




\section{Shabani-Fig.3}

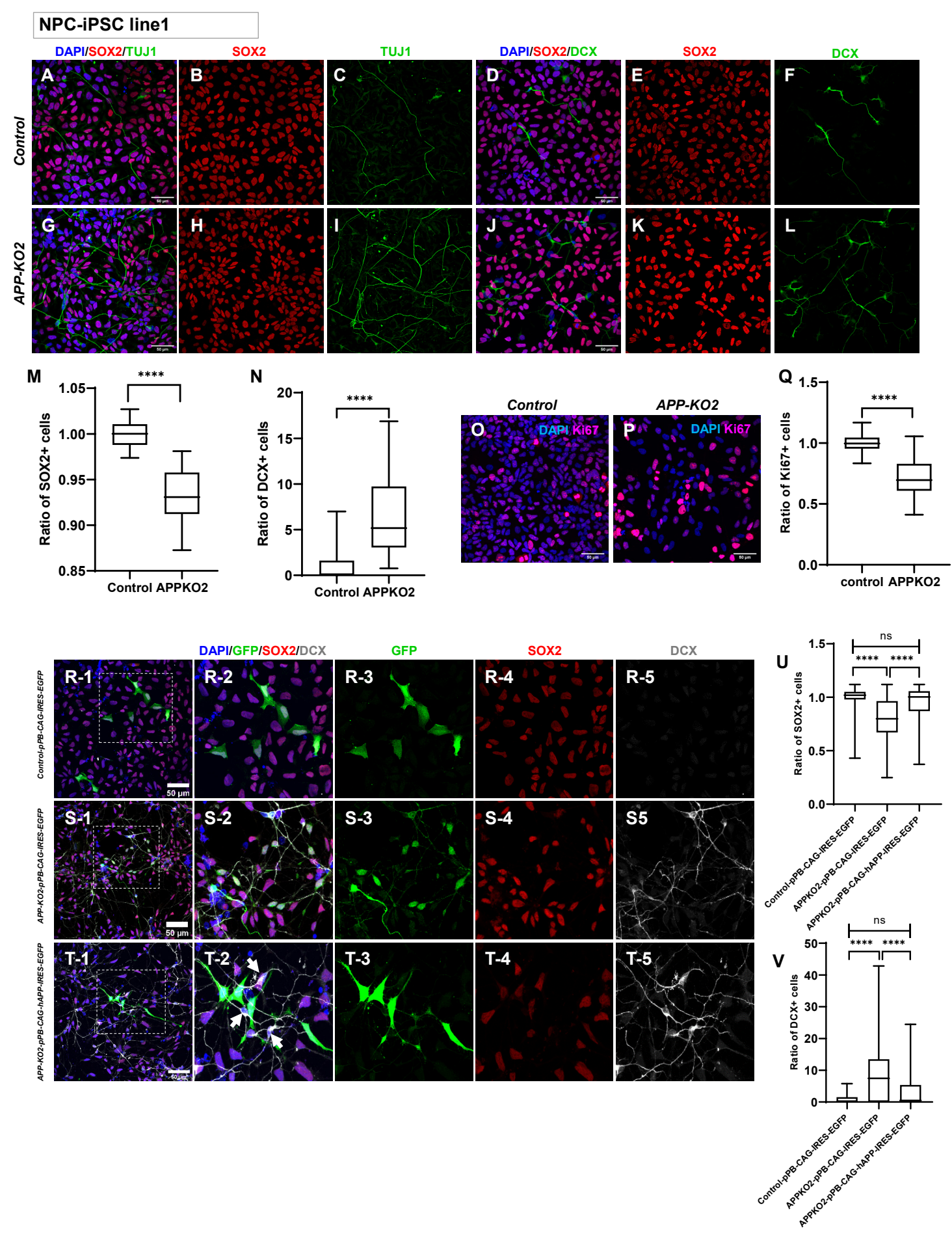

Fig. 3. Loss of APP drives increased differentiation of neural progenitors. a-l, NPCs from control and $A P P-K O 2$ stained for SOX2/TUJ1 and SOX2/DCX m, Significant decrease in ratio of SOX2+cells and $\mathbf{n}$, Significant increase in ratio of $\mathrm{DCX}+$ in $A P P-K O 2(\mathrm{n}=3$, unpaired t-test, $\mathrm{p}<0.0001$, scale bar $50 \mu \mathrm{m}) . \mathbf{0}-\mathbf{q}$, Significant decrease in ratio of Ki67+ cells in APP-KO2 $(\mathrm{n}=3$, 
unpaired t-test, $\mathrm{p}<0.0001$, scale bar $50 \mu \mathrm{m})$. r1-v, APP cell autonomously maintains NPCs in a progenitor stage. r1-r5, Control NPCs transfected with $p P B-C A G-I R E S-E G F P$ s1-s5, APP-KO2 NPCs transfected with $p P B-C A G-I R E S-E G F P$ t1-t5, $A P P-K O 2$ NPCs transfected with $p P B-C A G-$ $h A P P-I R E S-E G F P$ and stained for GFP/SOX2/DCX. APP rescued the phenotype of transfected cells but not neighboring cells (yellow arrows in $\mathrm{t} 2$ ). $\mathbf{u}$, Ratio of SOX $2+$ and $\mathbf{v}, \mathrm{DCX}+$ cells in all 3 conditions $(\mathrm{n}=2$, Ordinary one-way ANOVA, $\mathrm{p}<0.0001$, scale bar $50 \mu \mathrm{m})$. 

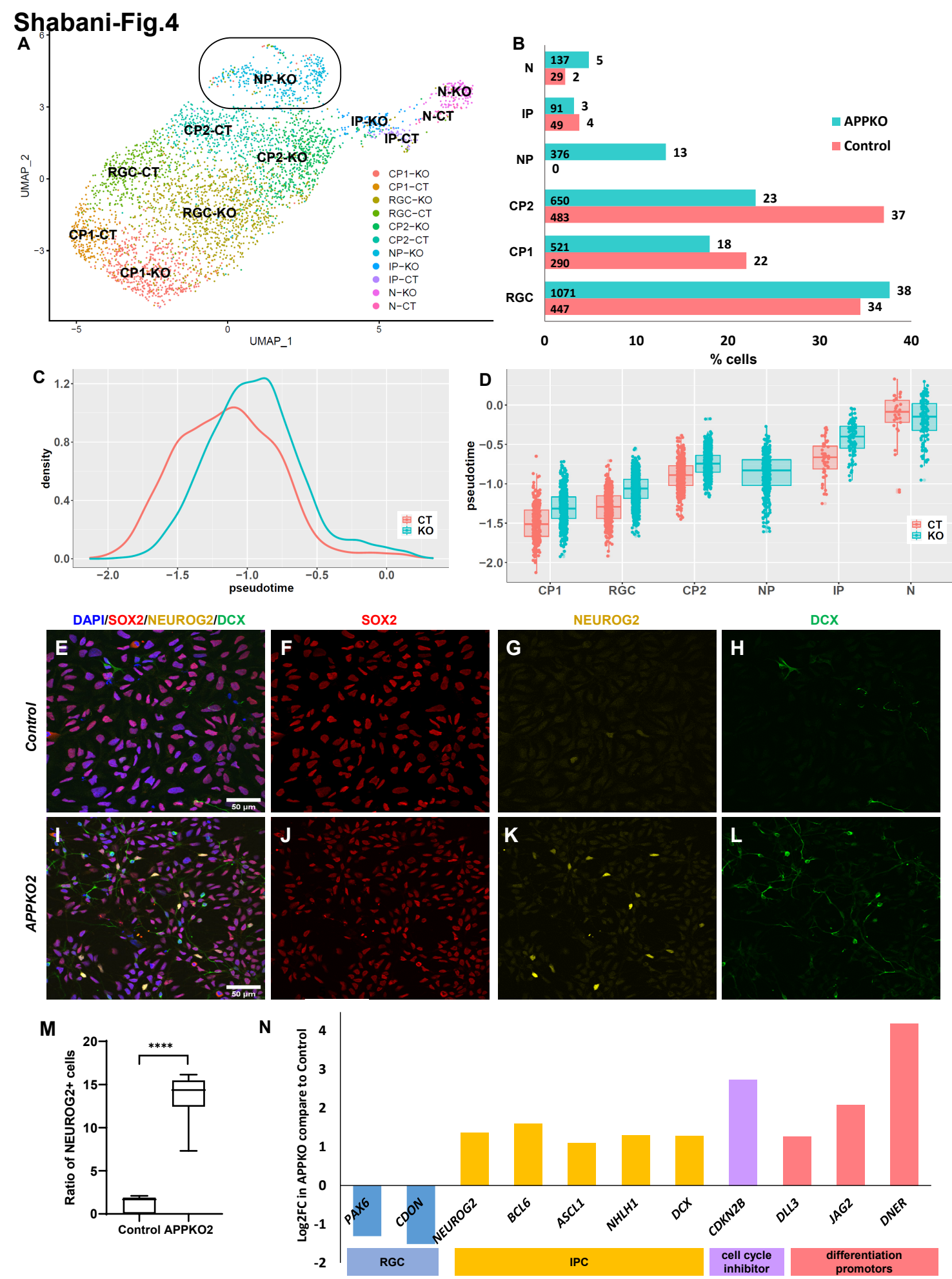

Fig. 4. Neurogenic progenitors explain the increased differentiation of $A P P-K O$ NPCs. a, Clusters of control and $A P P-K O 2 \mathrm{NPC}(\mathrm{CT}=$ control, $\mathrm{KO}=\mathrm{APPKO} 2)$. b, Emergence of a new "neurogenic progenitors" cluster in $A P P-K O 2$, number and percentage of cells in each cluster in control and $A P P-K O 2$ ( $\mathrm{RGC}=$ radial glial cell, $\mathrm{CP} 1=$ cycling progenitor1, $\mathrm{CP} 2=$ cycling 
progenitor2, $\mathrm{IP}=$ intermediate progenitor, $\mathrm{NP}=$ neurogenic progenitor, $\mathrm{N}=$ neuron). $\mathbf{c}-\mathrm{d}$, Pseudo time analysis show a shift toward neural fate in $A P P-K O$ clusters. e-l, Staining of control and $A P P-K O 2$ NPC for NEUROG2/SOX2/DCX. m, Ratio of NEUROG2+ cells is 15-fold higher in APP-KO2 (unpaired t-test, $\mathrm{p}<0.0001$ ) with the majority being SOX2+/NEUROG2+ (yellow arrow in $\mathrm{i}-\mathrm{j}$ ). Some DCX+ cells are also positive for SOX2 (green arrows in j-k). n, Bulk RNAseq shows downregulation of RGC markers (PAX6 and CDON) and upregulation of neurogenic genes (NEUROG2, BCL6, ASCL1, NHLH1, and DCX), cell cycle inhibitor (CDKN2B) and differentiation promotors Notch ligands DLL3, JAG2, DNER. 


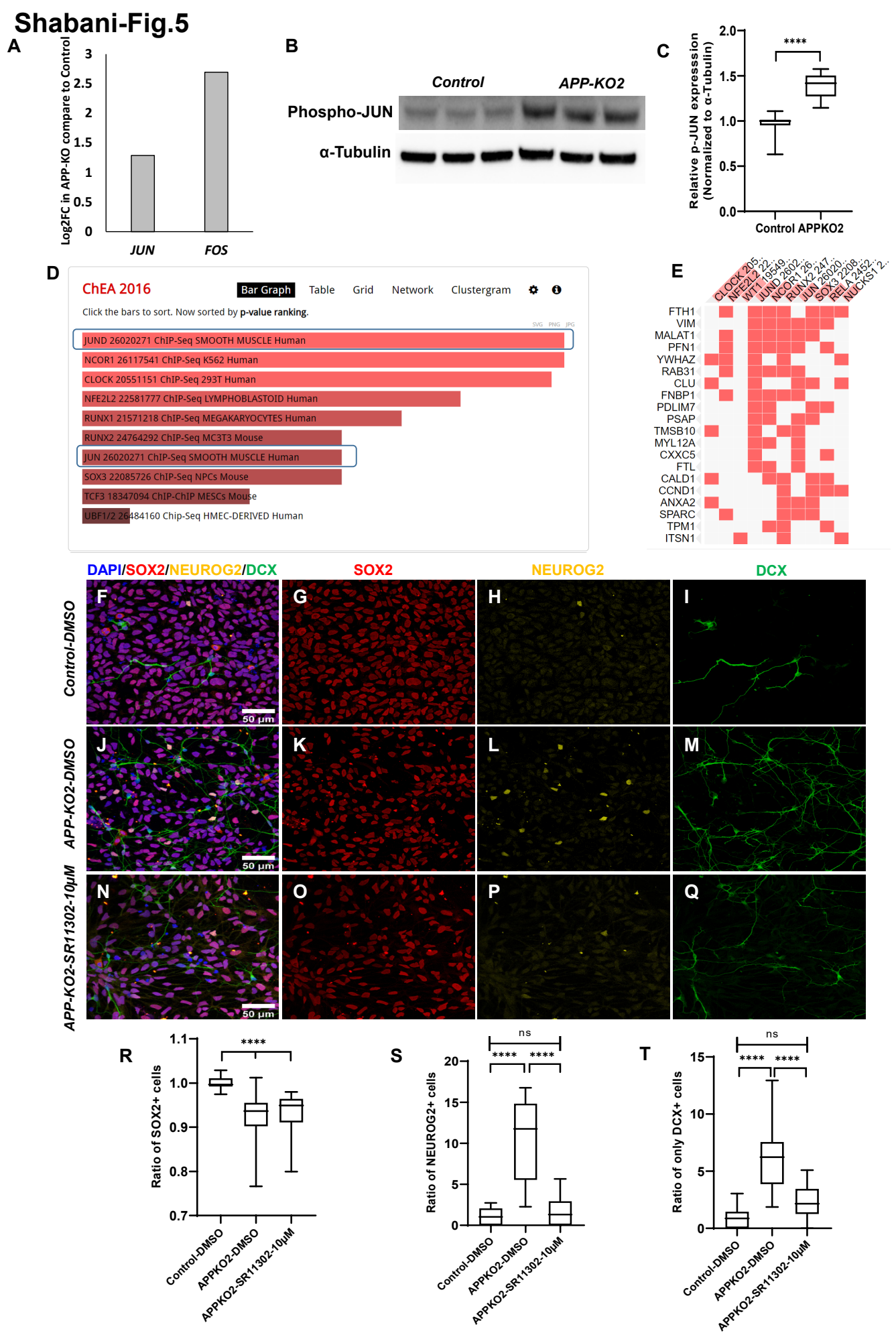

Fig. 5. Loss of APP impacts NPC differentiation through AP1. A, Upregulation of JUN and FOS in APP-KO2 NPC in bulk RNA sequencing results. B-C, Western blot shows significant increase in phospho-JUN in APP-KO2 NPC compared to control ( $\mathrm{n}=3$ biologically independent repeats, unpaired t-test, $\mathrm{P}<0.0001)$. D-E, JUN family members bind to marker genes of neurogenic 
progenitors (NPs) in the ChEA 2016 CHIP-seq database. F-Q, NPC treated with AP inhibitor (SR11302) and stained for SOX2/DCX/NEUROG2 showed $\mathbf{R}$, no rescue of ratio of SOX2+ in $A P P-K O 2$ treated with SR11302 compared to untreated $A P P-K O 2$ NPC (n=3, Ordinary one-way ANOVA, $\mathrm{p}<0.0001$ for control-DMSO vs $A P P-K O 2-\mathrm{DMSO}$ and $A P P-K O 2-\mathrm{SR} 11302)$. S, Significant decrease in NEUROG2+ cells in $A P P-K O 2$ treated with SR11302 compared to untreated $A P P-K O 2 \mathrm{NPC}$ (n=3, Ordinary one-way ANOVA, $\mathrm{p}<0.0001$ for control-DMSO vs $A P P$ $K O 2-\mathrm{DMSO}$ and $A P P-K O 2-\mathrm{DMSO}$ vs $A P P-K O 2-\mathrm{SR} 11302)$. T, Significant decrease in DCX+ cells in $A P P-K O 2$ treated with SR11302 compared to untreated $A P P-K O 2$ NPC (n=3, Ordinary one-way ANOVA, $\mathrm{p}<0.0001$ for control-DMSO vs $A P P-K O 2-\mathrm{DMSO}$ and $A P P-K O 2-\mathrm{DMSO}$ vs $A P P$-KO2-SR11302). Scale bar $50 \mu \mathrm{m}$ for all the images. 


\section{Shabani-Fig.6}

A
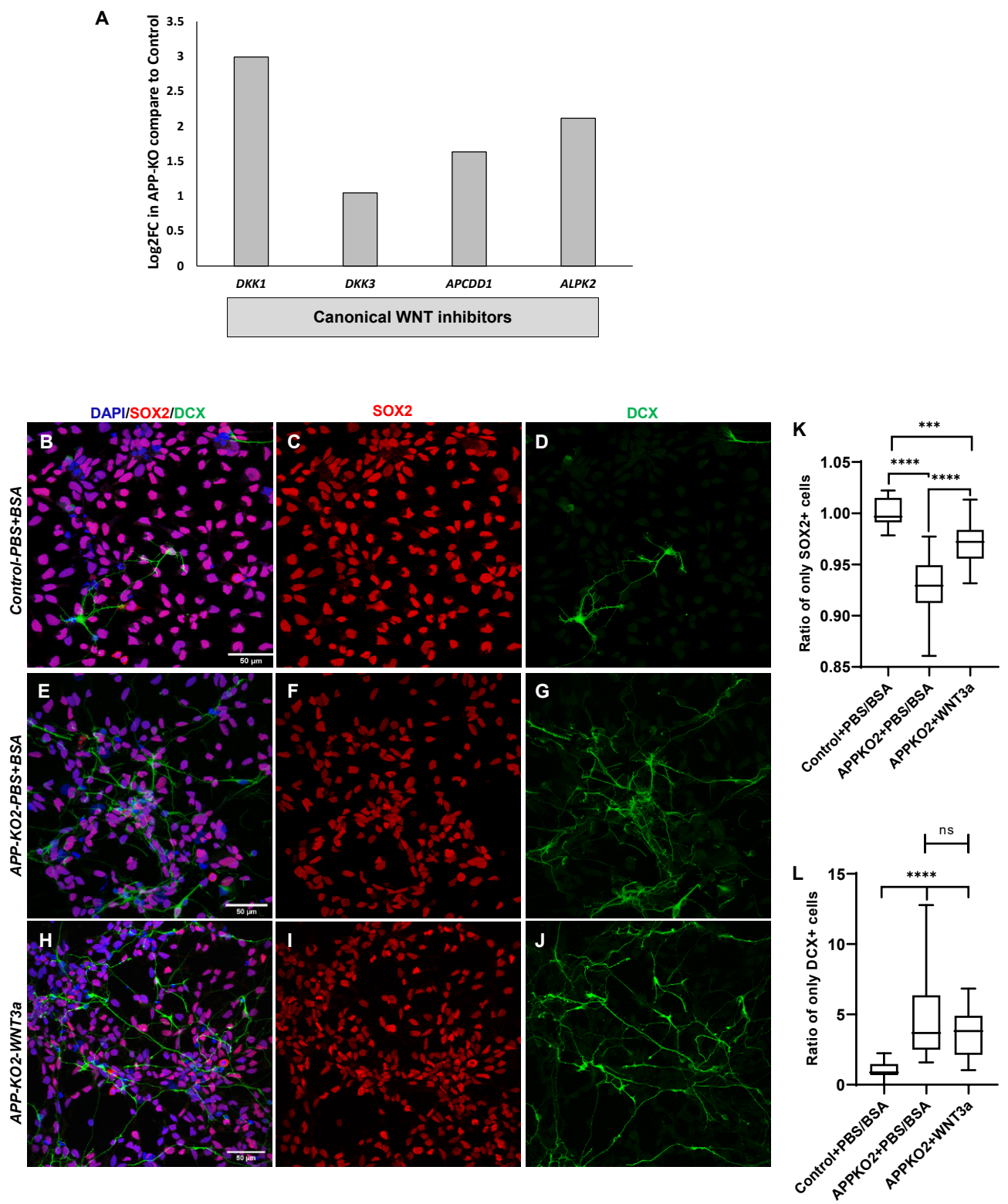

Fig. 6. Loss of APP attenuates progenitor state thorough canonical WNT signaling. A, Upregulation of canonical WNT inhibitors DKK1, DKK3, APCDD1, and ALPK2 in APP-KO2 NPC compared to control. B-J, APP-KO2 NPC treated with canonical WNT ligand (WNT3a) and stained for SOX2 and DCX. K, Ratio of SOX2+ was significantly rescued in APP-KO2 NPC treated with WNT3a ( $\mathrm{n}=3$, Ordinary one-way ANOVA, $\mathrm{p}<0.0001$ for control-PBS+BSA vs $A P P$ $K O 2-\mathrm{PBS}+\mathrm{BSA}$ and $A P P-K O 2-\mathrm{PBS}+\mathrm{BSA}$ vs $A P P-K O 2-\mathrm{WNT} 3 \mathrm{a}, \mathrm{p}=0.0002$ for control vs $A P P$ $K O 2-W N T 3 a)$. L, Ratio of DCX+ cells was not rescued after treatment with WNT3a (n=3, Ordinary one-way ANOVA, $\mathrm{p}<0.0001$ for control-PBS+BSA vs APP-KO2-PBS+BSA and control vs $A P P-K O 2-W N T 3 a)$. 


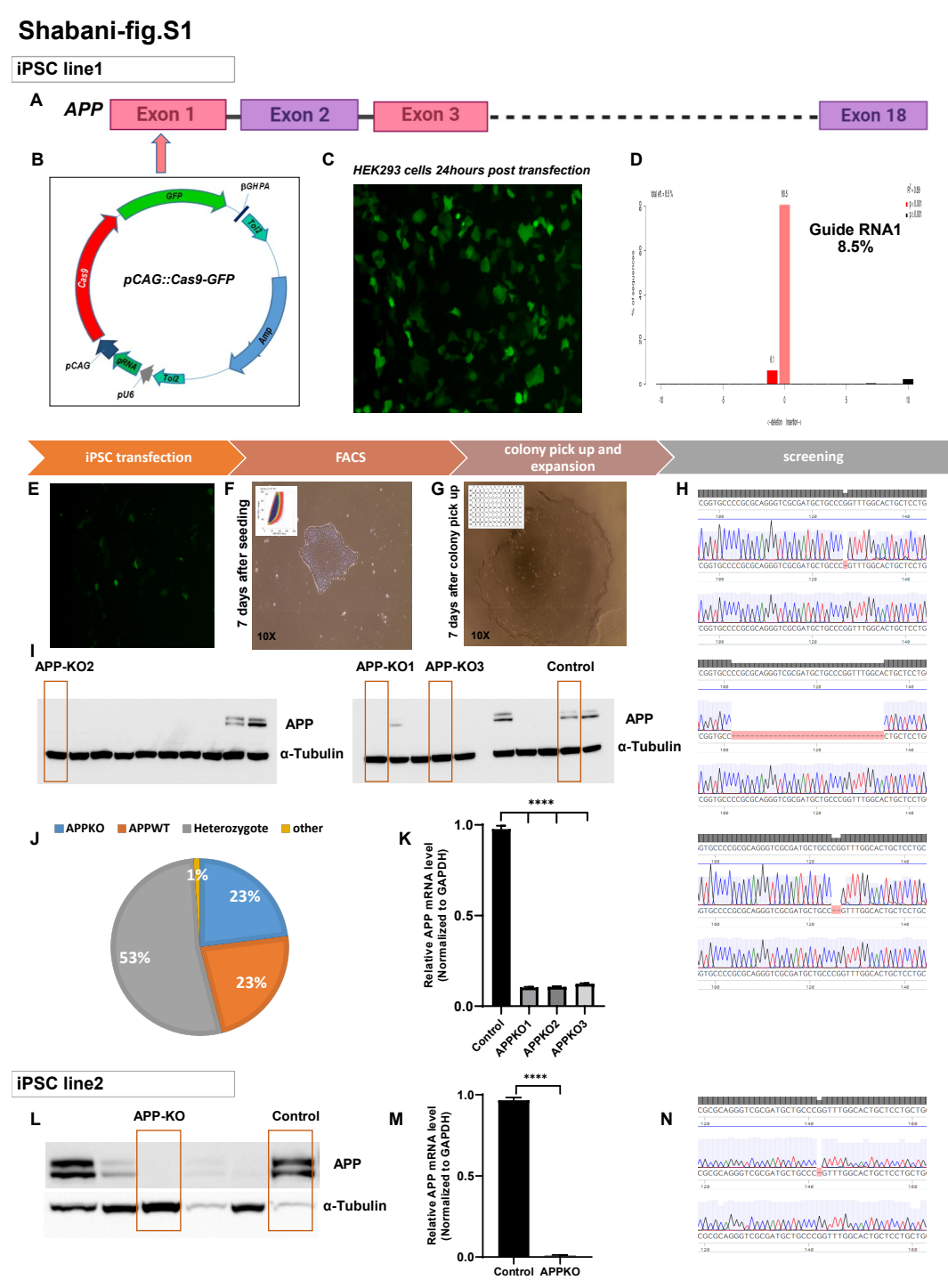

Fig. S1. Generation of iPSC derived APP knockout clones. A-K, Individual steps in generating $A P P$ knock out from iPSC line1. A, Targeting exon1 of $A P P$ by B, Plasmid vector containing guide RNA, CAS9 and GFP. C, HEK293 cell 24 hours after transfection. D, Cleavage efficiency of Guide RNA1 that was used for transfection of iPSC. E-H, iPSC transfection by Guide RNA1, post FACS morphology and sequencing results of clones. I, Western blot results, which confirm no expression of APP protein in 14 out of 60 clones. Control and three $A P P-K O$ clones were chosen for further experiments. $\mathbf{J}$, Distribution of clones with different $A P P$ genotypes $(23 \%=A P P-K O, 23 \%=A P P W T$ and $53 \%=$ heterozygote and $1 \%=$ other mutation). $\mathbf{K}$, qPCR confirms low level of $A P P$ mRNA expression in $A P P-K O$ clones. L-N, Generation of $A P P-K O$ clones from iPSC line 2 and confirming no expression of APP protein. 


\section{Shabani-fig.S2}

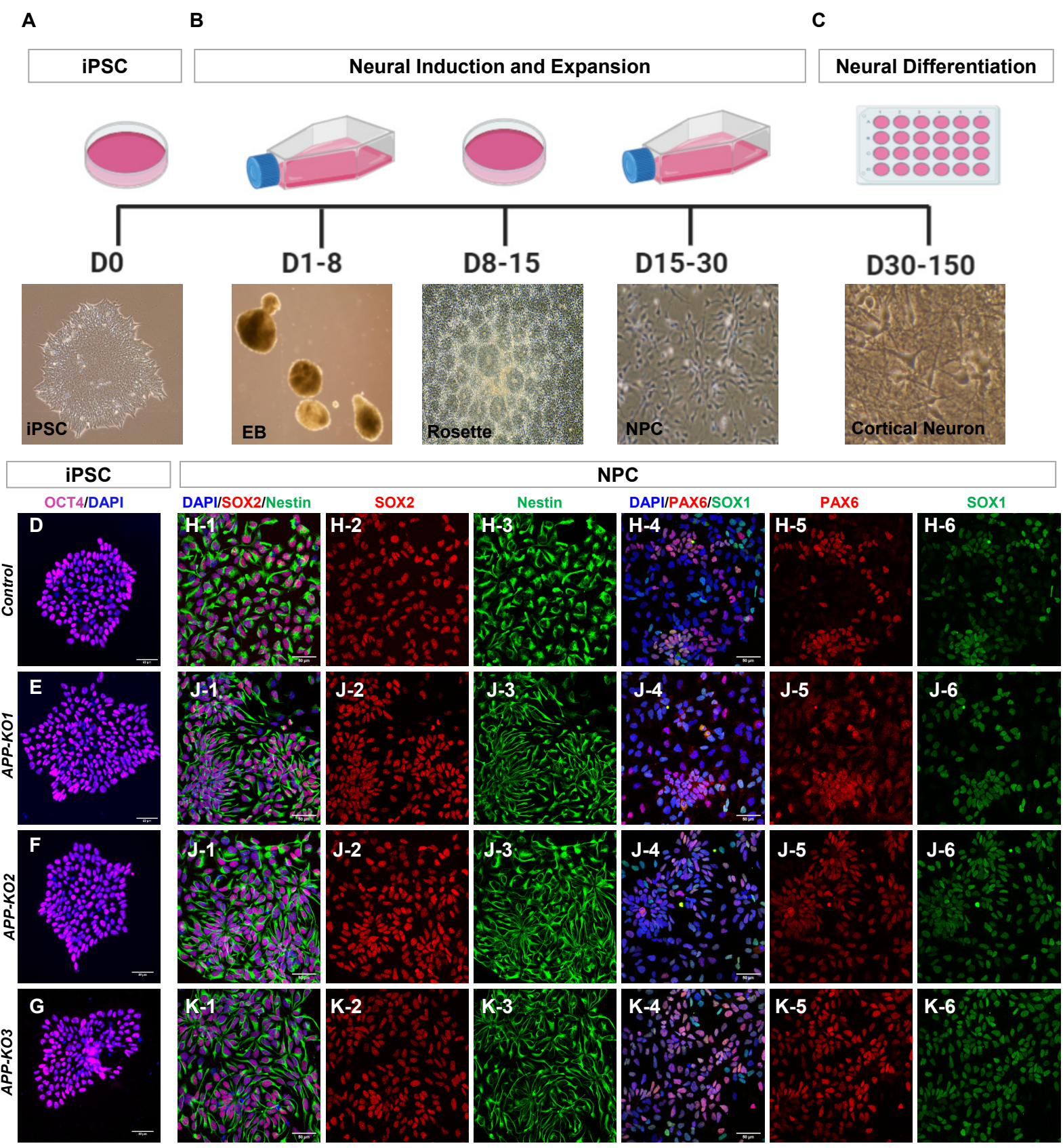

Fig. S2. Cortical differentiation protocol and characterization of neural precursor cells. A-C, Schematic showing different steps in generating cortical neurons. D-G, Confirming pluripotency of control and APP-KOs iPSCs with pluripotency marker OCT4. H1-K6, Characterization of control and APP-KOs derived neural precursor cells with

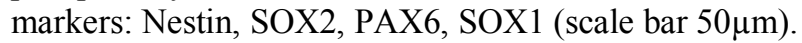




\section{Shabani-fig.S3}

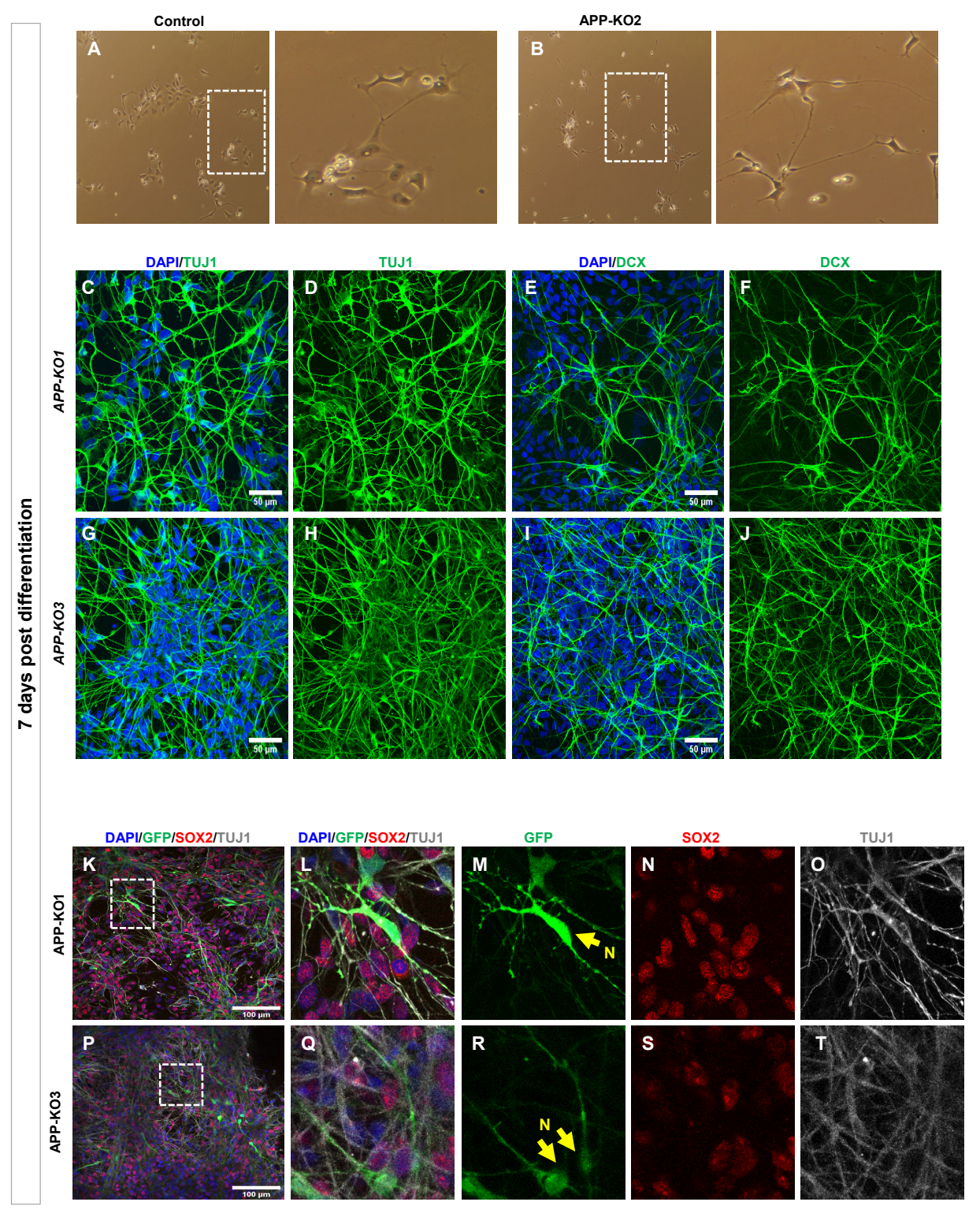

Fig. S3. Increased differentiation of $A P P-K O 1$ and $A P P-K O 3$ neural precursor cells within 7 days post differentiation. A,B, Morphological changes 7 days post differentiation neurons for control and $A P P-K O 2$. C-J, Appearance of neural markers TUJ1 and DCX occurring earlier in $A P P-K O 1$ and $A P P-K O 3$ compared to control 7 days post differentiation (Scale bar $50 \mu \mathrm{m}$ ). K-T, Expression of SOX2 and TUJ1 within GFP + cells in $A P P-K O 1$ and APP-KO3 (Scale bar $100 \mu \mathrm{m}, \mathrm{N}=$ neuron, $\mathrm{P}=$ Progenitor). 


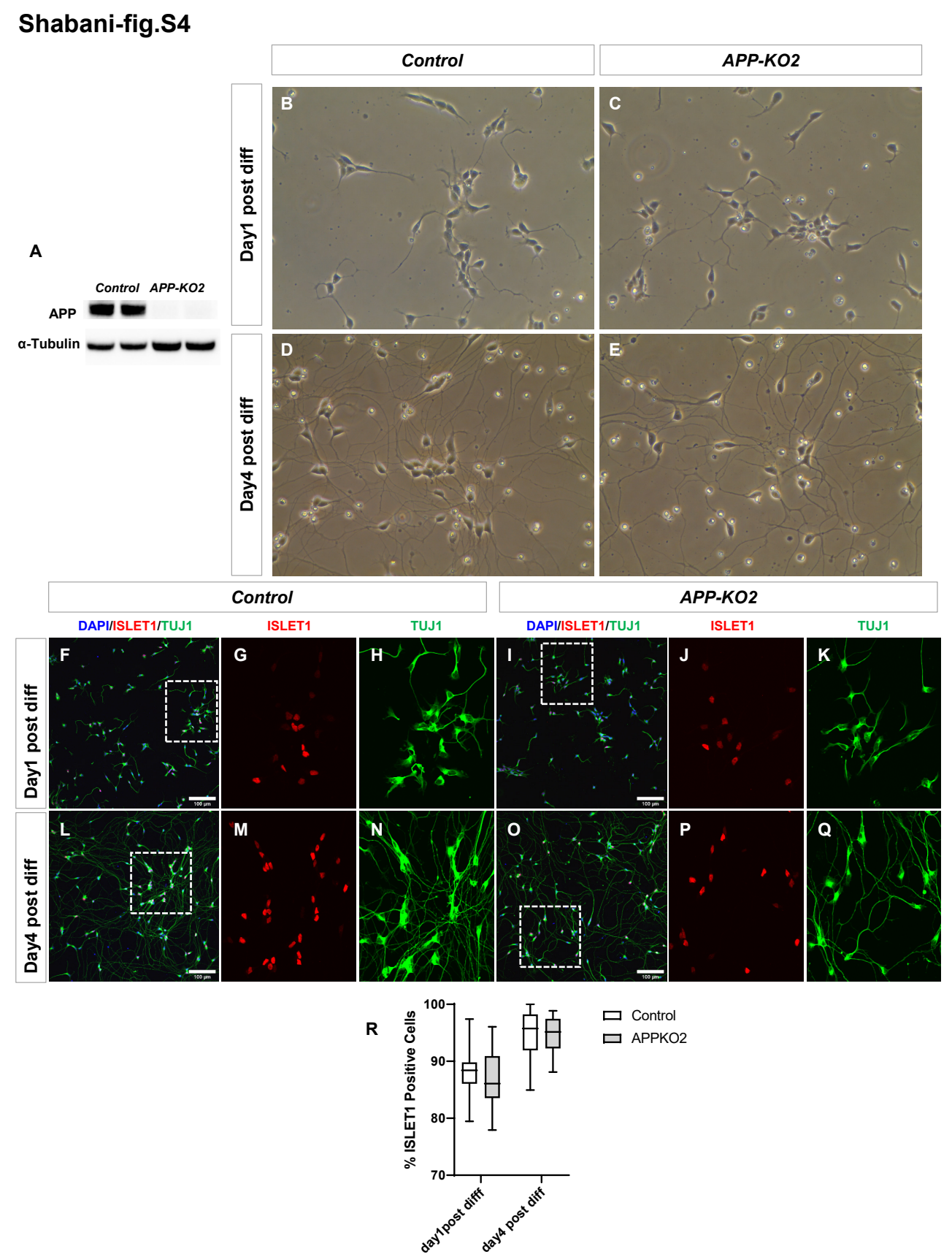

Fig. S4. No differences in ISLET1 motor neurons derived from control and APP-KO2. A, APP expression was confirmed in control by western blot in motor neuron precursor cells. B-E, Morphological changes in 1 day and 4 days post differentiation motor neurons. F-Q, 1 day and 4 days post differentiation motor neuron stained for ISLET1 and

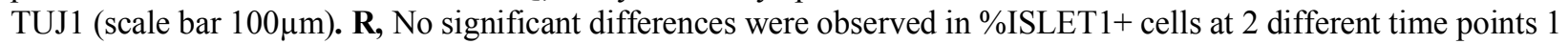
day and 4 days post differentiation ( $\mathrm{n}=3$ biological independent repeat, 2 way ANOVA). 


\section{Shabani-fig.S5}

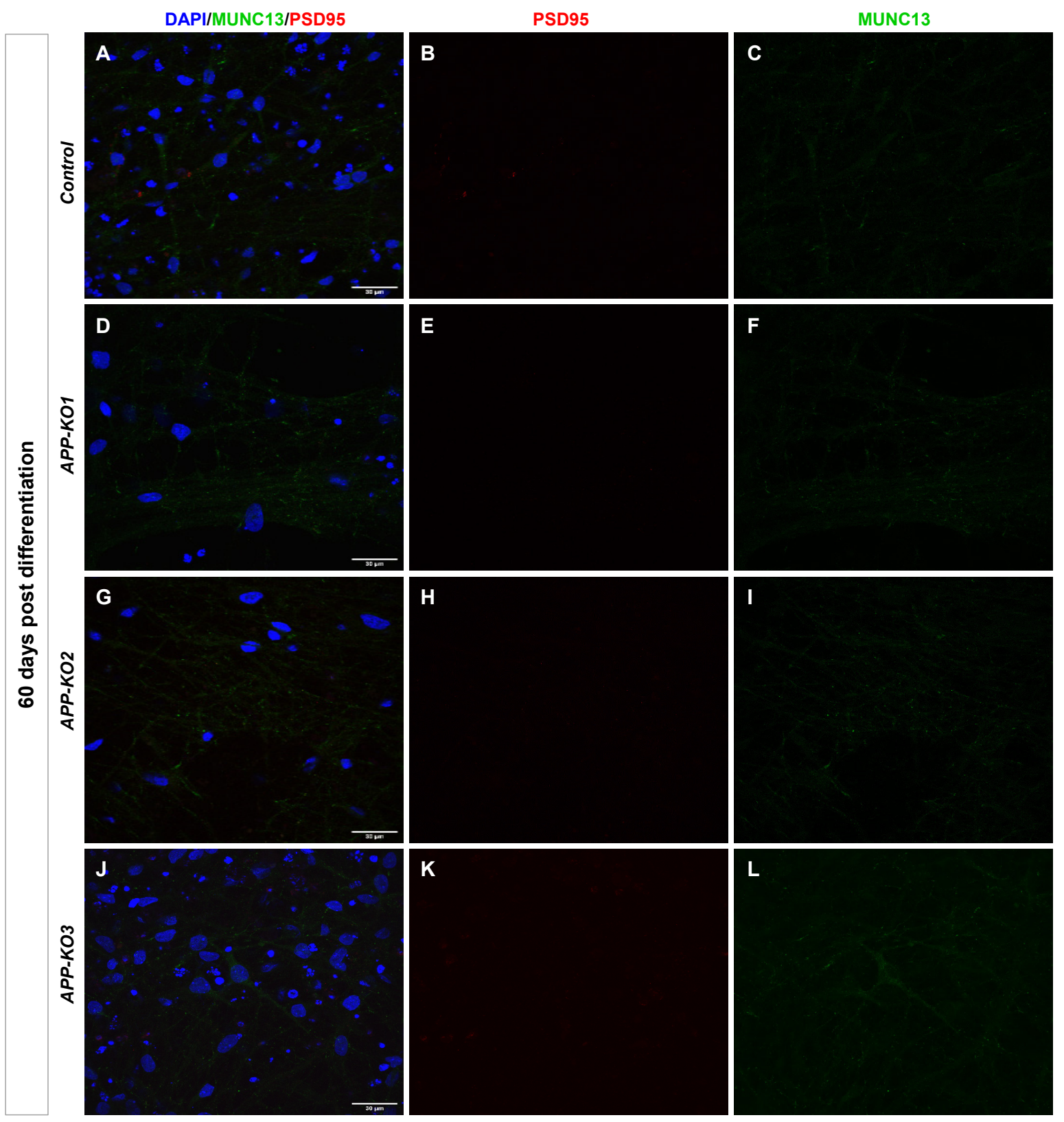

Fig. S5. Presynaptic markers begin to be expressed 60 days post differentiation in both $A P P-K O$ and controls. A-L, Weak MUNC13 (presynaptic marker) in control and all $3 A P P-K O s$, while no expression of PSD95 (post synaptic marker) expression was detected in either condition at this stage (scale bar $30 \mu \mathrm{m}$ ). 


\section{Shabani-fig.S6}

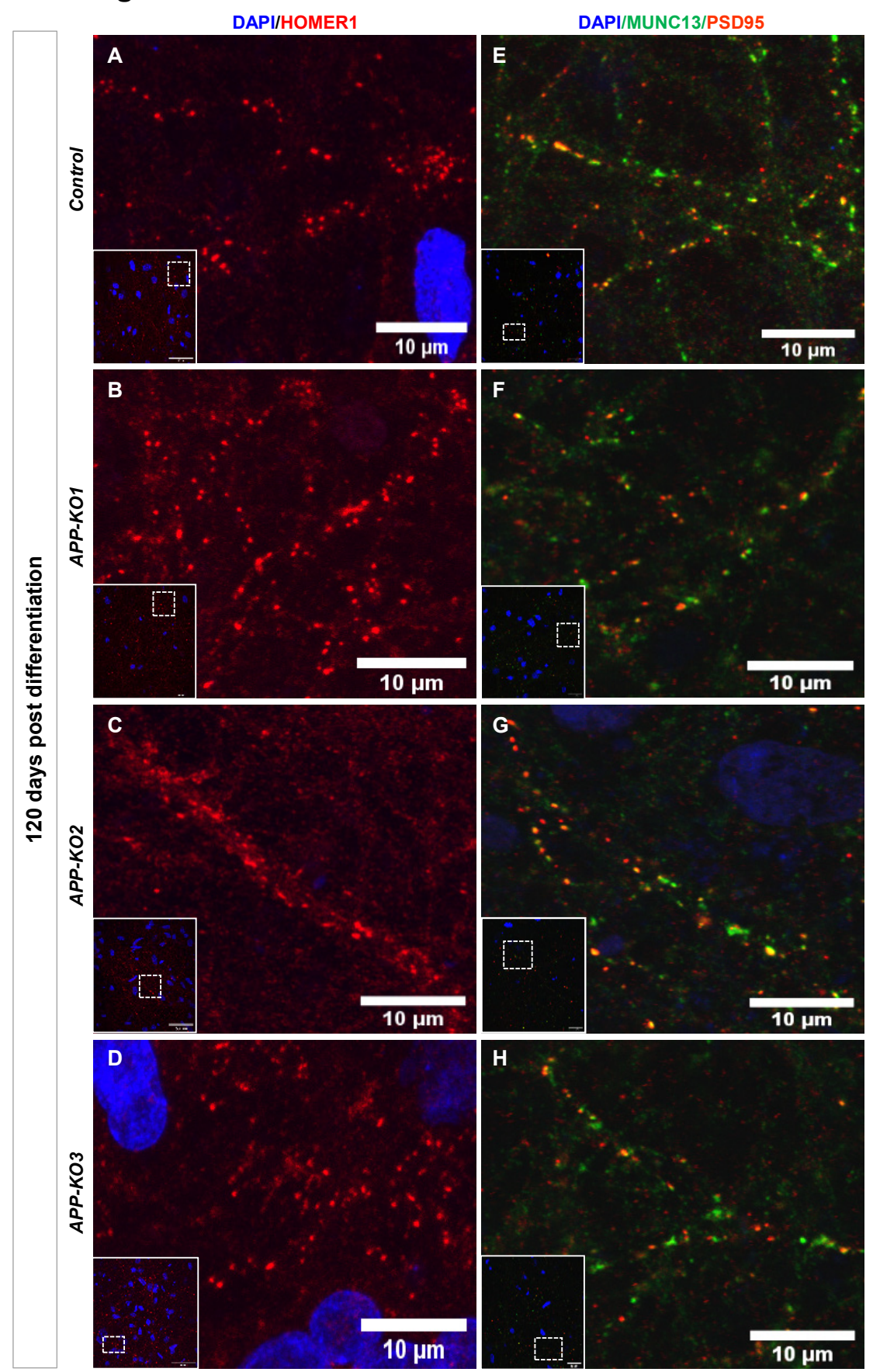

Fig. S6. Pre- and post- synaptic markers are expressed 120 days post differentiation in both $A P P-K O$ and controls. A-D, Homer 1(synapse marker of glutamatergic neuron), E-H, MUNC13 (presynaptic marker) and PSD95 (post synaptic marker) show the same pattern 120 days post differentiation in controls and three APP-KOs (scale bar $10 \mu \mathrm{m})$ 


\section{Shabani-fig.S7}
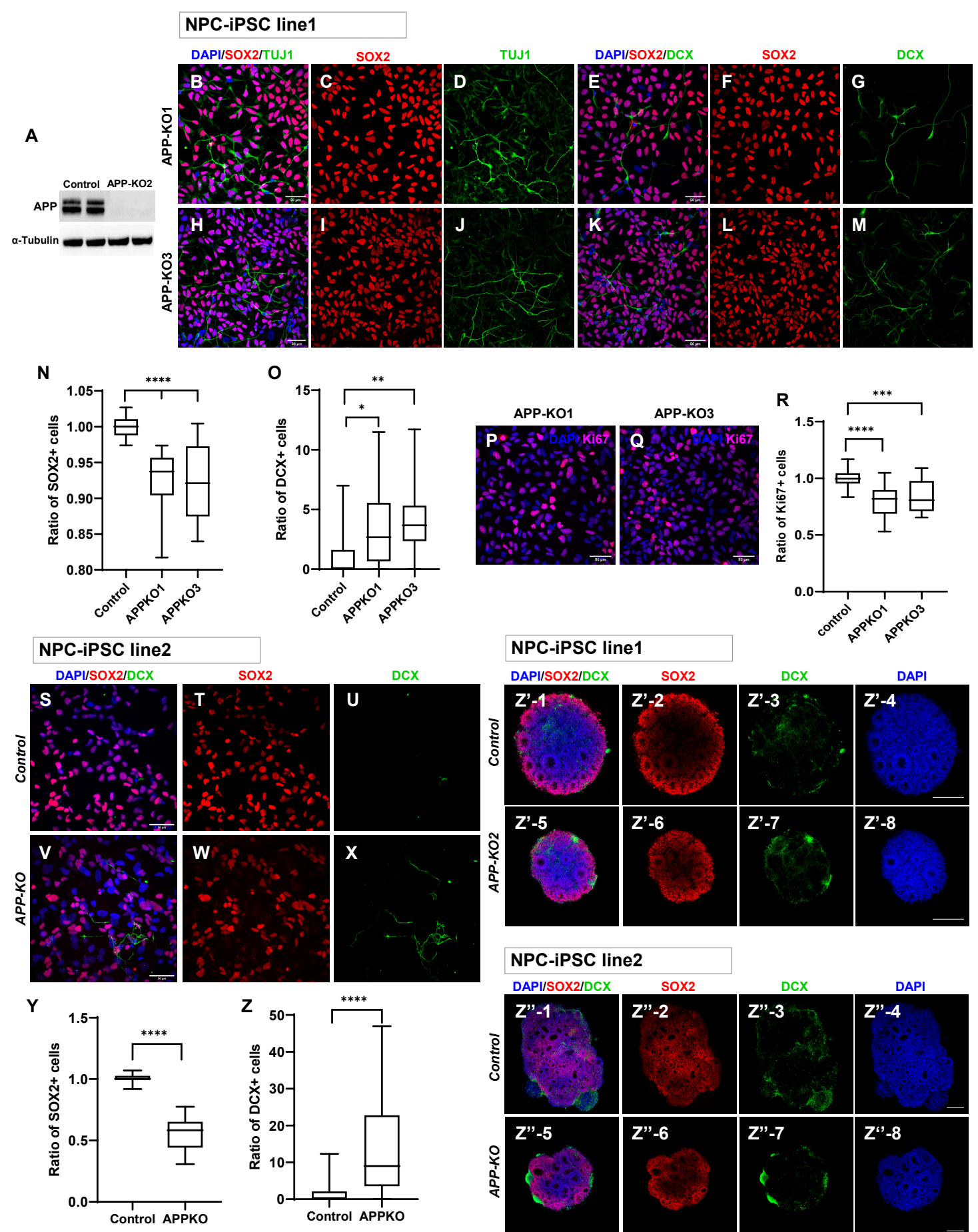

sox2
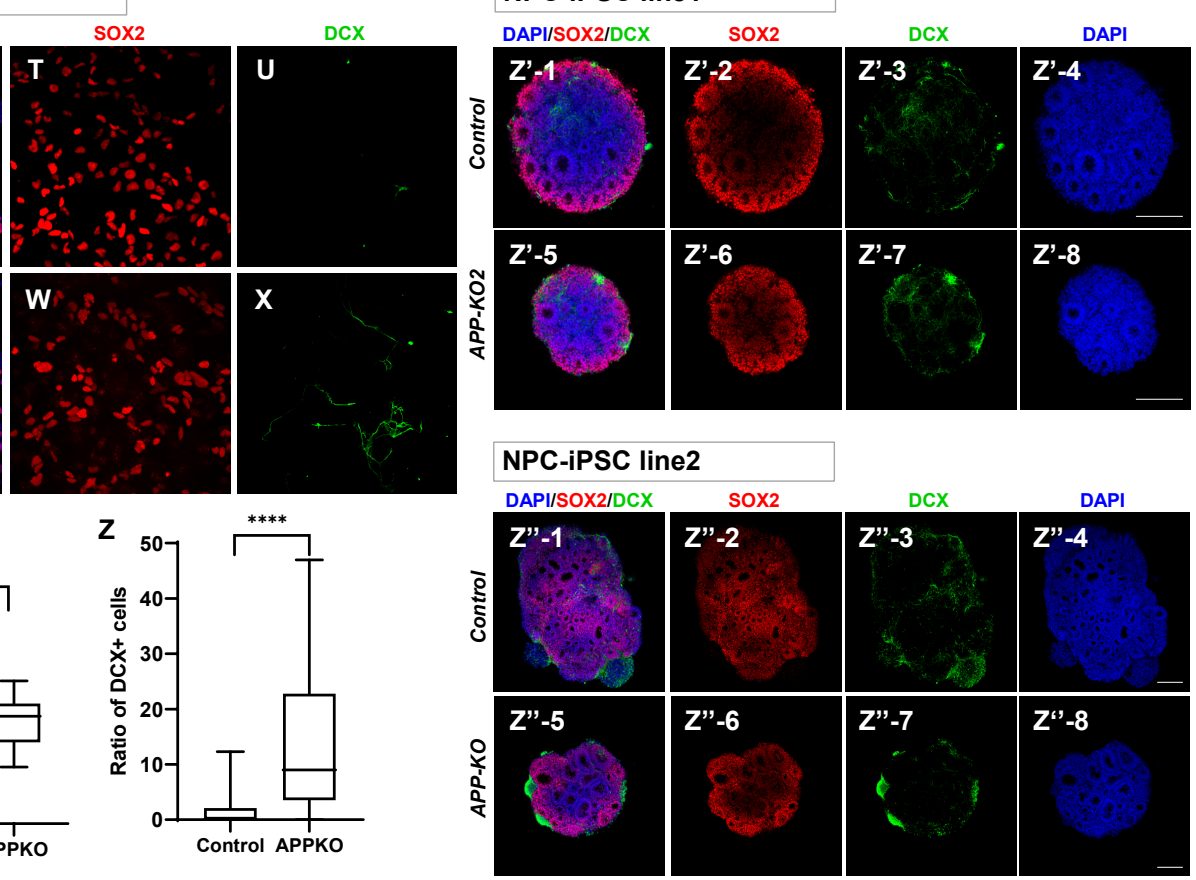

Fig. S7. Loss of APP causes increased differentiation in 3D organoids independent of genetic background. A, APP expression in control was confirmed in NPC by western blot. B-M, NPCs from $A P P-K O 1$ and $A P P-K O 3$ stained for SOX2/TUJ1 and SOX2/DCX and $\mathbf{N}$, Quantification of SOX2+ cells and $\mathbf{O}, \mathrm{DCX}+$ in neural precursor cells $(\mathrm{n}=3$, ordinary one-way ANOVA, $\mathrm{p}<0.0001$ for SOX2, $\mathrm{p}=0.0183$ and $\mathrm{p}=0.0038$ for DCX of APP-KO1 and APP-KO3, respectively. scale bar $50 \mu \mathrm{m})$. P-R, $A P P-K O 1$ and $A P P-K O 3 \mathrm{NPC}$ stained for Ki67 and quantification of that (n=3, ordinary one-way ANOVA, $\mathrm{p} \leq 0.0001$, scale bar $50 \mu \mathrm{m})$. S-Z, The results were reproducible in iPSC line $2(\mathrm{n}=3$, unpaired t-test, $\mathrm{p}<0.0001$, scale bar $50 \mu \mathrm{m})$. Z' $\mathbf{1}-\mathbf{Z}$ '8, Cortical organoid for control and $A P P-K O 2$ from iPSC line 1 
bioRxiv preprint doi: https://doi.org/10.1101/2021.02.17.431707; this version posted February 17, 2021. The copyright holder for this preprint (which was not certified by peer review) is the author/funder. All rights reserved. No reuse allowed without permission.

stained for SOX2/DCX (n=3 organoids, scale bar 100 $\mu \mathrm{m}$ ). Z"1-Z"8, Cortical organoid for control and APP-KO from iPSC line 2 stained for SOX2/DCX ( $\mathrm{n}=3$ organoids, scale bar $200 \mu \mathrm{m})$, showing clusters of more intense DCX+ cells. 


\section{Shabani-fig.S8}
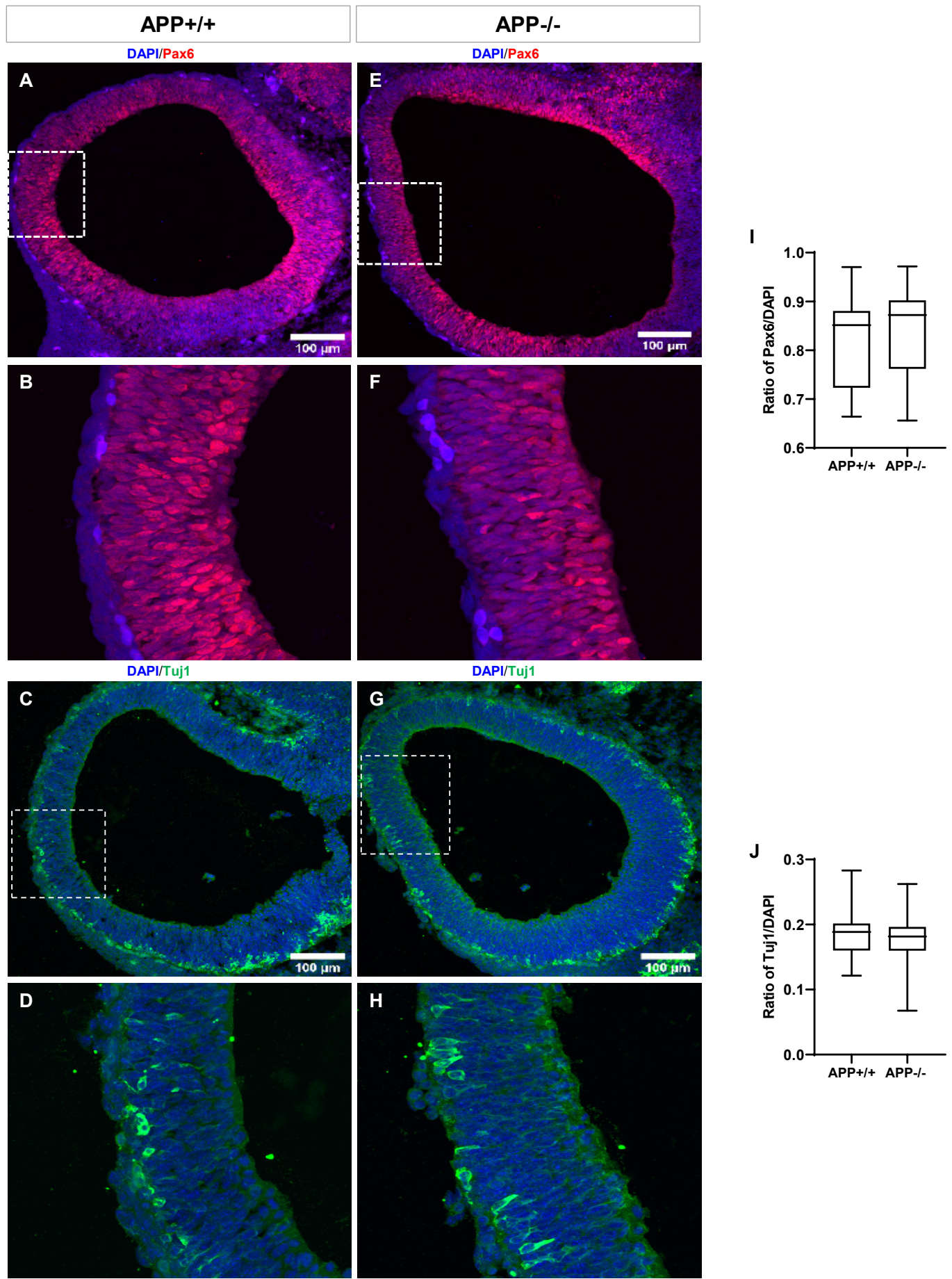

Fig. S8. No difference in Pax6 and Tuj1 expression was observed in in $A P P+/+$ and $A P P-/-$ E10.5 mouse embryos. A-H, Brain sections of E10.5 embryo stained for Pax6 and Tuj1. I-J, No difference was observed in the ratio of Pax6/DAPI and Tuj1/DAPI in $A P P+/+$ and $A P P-/-$ embryo ( $\mathrm{n}=3$ embryos, unpaired t-test). 


\section{Shabani-fig. S9}
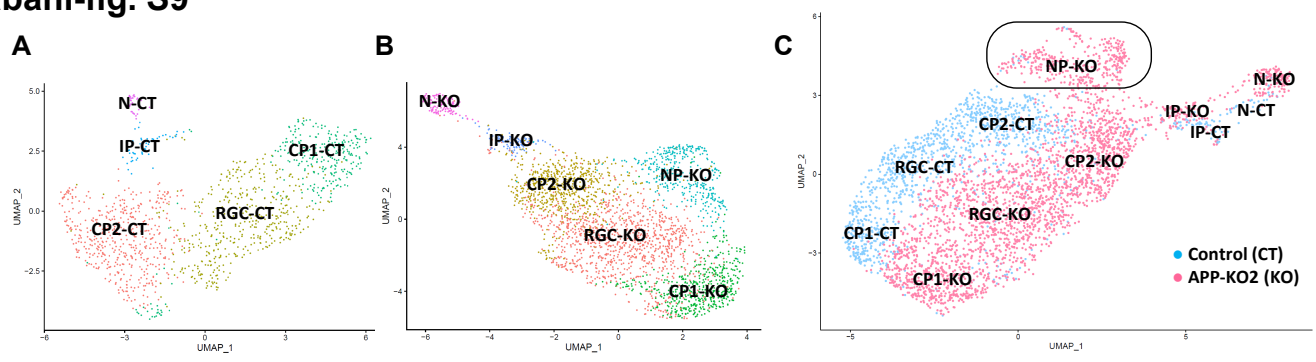

D

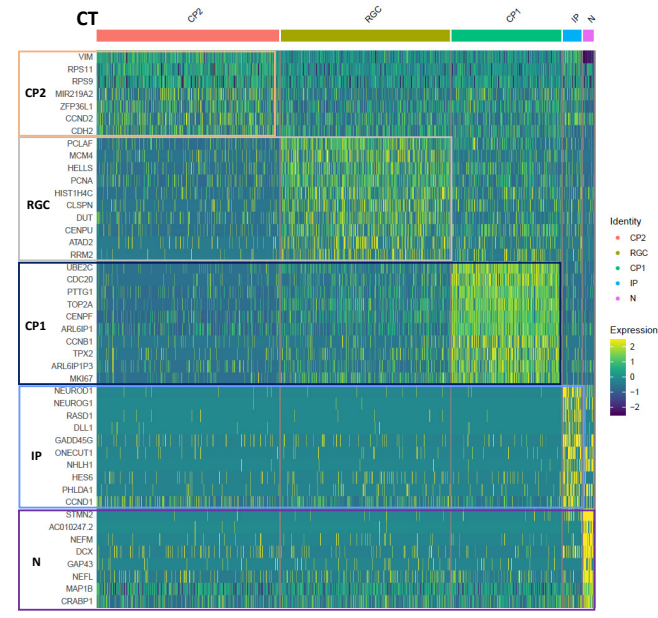

$\mathrm{F}$

E
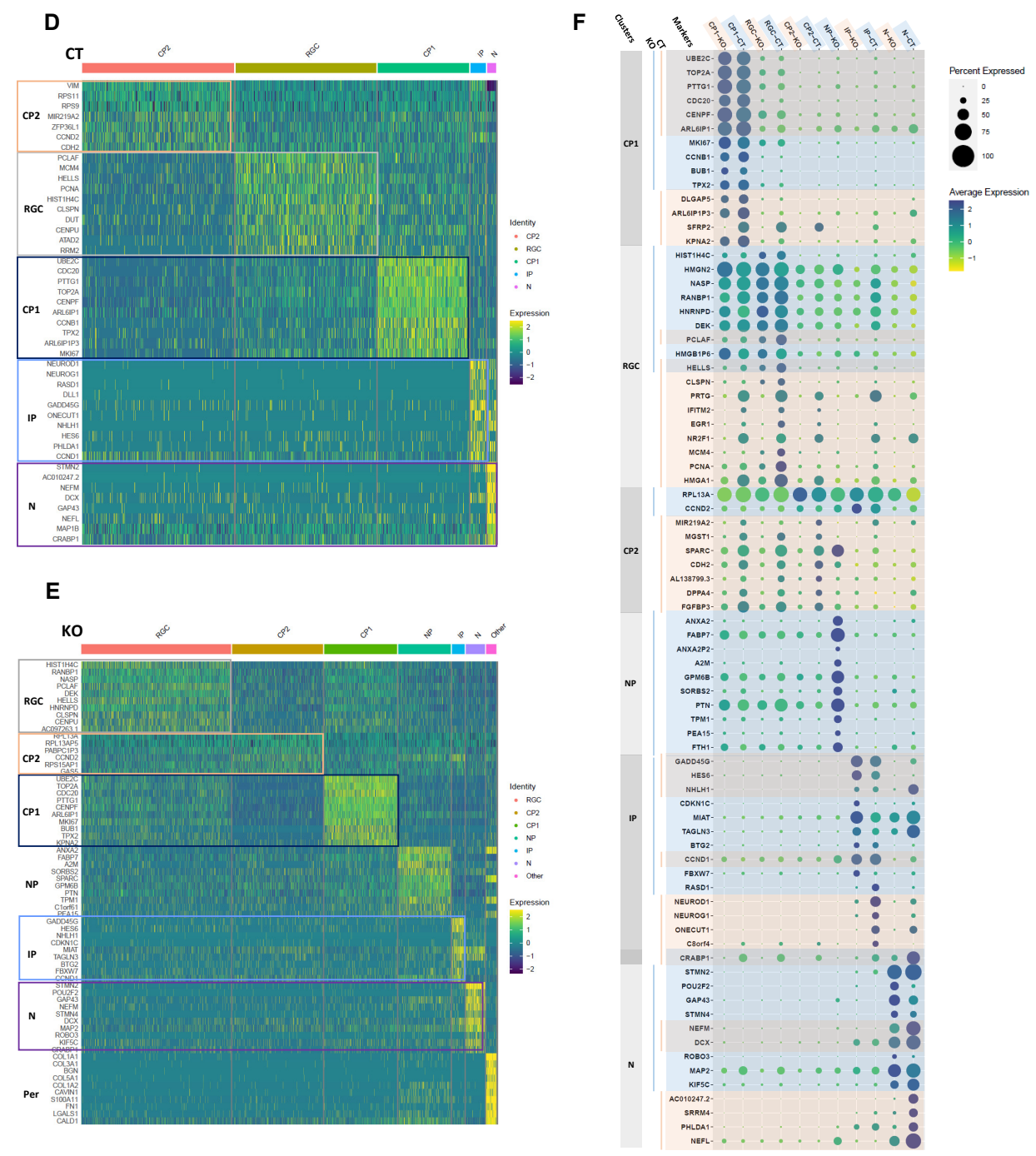

Fig. S9. Heat maps of different clusters from single cell RNA sequencing. A-C, UMAP of different clusters in A, control B, $A P P-K O 2$ and $\mathbf{c}$, control and $A P P-K O 2$ together. Heat maps of $\mathbf{D}$, five clusters in control and $\mathbf{E}$, seven clusters in $A P P-K O 2$. F, Representation of the analysis of expression levels and frequency of the marker genes that identify the various clusters control and $A P P K O 2(\mathrm{CT}=$ control, $\mathrm{KO}=A P P-K O 2)$. Color indicates expression level while the size of the circle indicates $\%$ of cells expressing the marker. 


\section{Shabani-fig.S10}
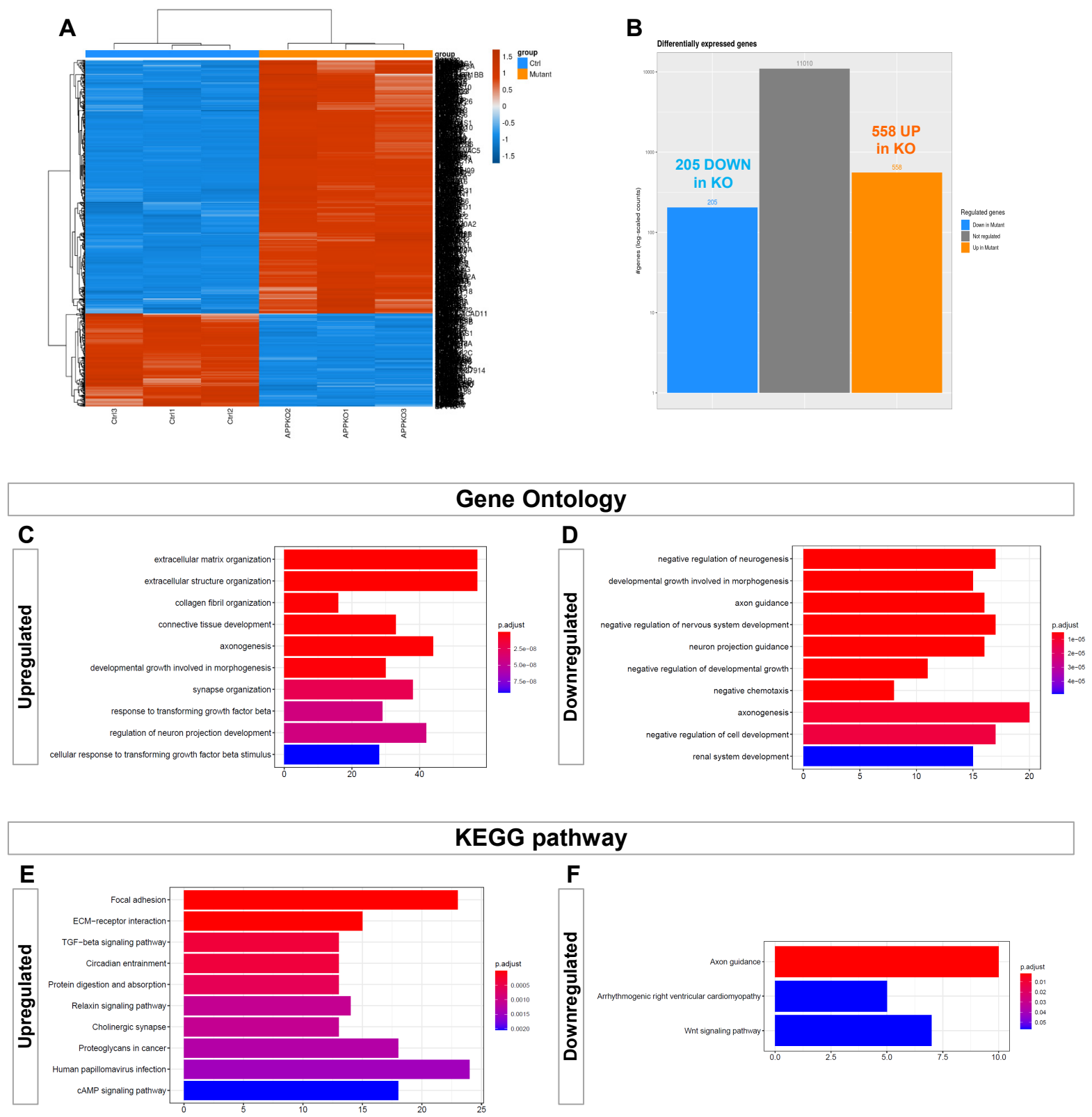

Fig. S10. Gene ontology and pathway analysis for upregulated and downregulated genes. A, Heat map of differentially expressed genes shows strikingly different patterns in control and APP-KO2 NPC. (ctrl1,2,3 correspond to 3 technical repeats of control and $A P P-K O 1,2,3$ correspond to 3 technical repeats of $A P P-K O 2$ ). B, 763 differentially expressed genes in $A P P-K O 2$ NPC compared to control which 558 genes are upregulated and 205 gens are downregulated. C-D, Gene ontology E-F, Pathway analysis of upregulated and downregulated genes. 